\title{
Black hole accretion and host galaxies of obscured quasars in XMM-COSMOS ${ }^{\star}$
}

\author{
V. Mainieri ${ }^{1}$, A. Bongiorno ${ }^{2}$, A. Merloni ${ }^{2,3}$, M. Aller ${ }^{4}$, M. Carollo $^{4}$, K. Iwasawa ${ }^{5}$, A. M. Koekemoer ${ }^{6}$, M. Mignoli ${ }^{7}$,

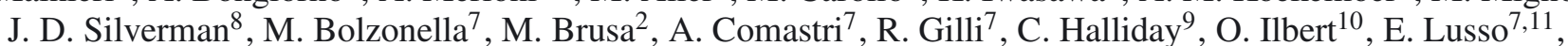 \\ M. Salvato ${ }^{12}$, C. Vignali $^{11}$, G. Zamorani ${ }^{7}$, T. Contini ${ }^{13}$, J.-P. Kneib ${ }^{10}$, O. Le Fèvre ${ }^{10}$, S. Lilly ${ }^{4}$, A. Renzini ${ }^{14}$, \\ M. Scodeggio ${ }^{15}$, I. Balestra ${ }^{2}$, S. Bardelli ${ }^{7}$, K. Caputi ${ }^{16}$, G. Coppa ${ }^{7}$, O. Cucciati ${ }^{15}$, S. de la Torre ${ }^{16}$, L. de Ravel ${ }^{10}$, \\ P. Franzetti1 ${ }^{15}$, B. Garilli ${ }^{15}$, A. Iovino ${ }^{17}$, P. Kampczyk ${ }^{4}$, C. Knobel ${ }^{4}$, K. Kovač ${ }^{18}$, F. Lamareille ${ }^{13}$, J.-F. Le Borgne ${ }^{13}$, \\ V. Le Brun ${ }^{10}$, C. Maier ${ }^{4}$, P. Nair ${ }^{7}$, R. Pello ${ }^{13}$, Y. Peng ${ }^{4}$, E. Perez Montero ${ }^{13}$, L. Pozzetti ${ }^{7}$, E. Ricciardelli ${ }^{19}$, M. Tanaka ${ }^{8}$,

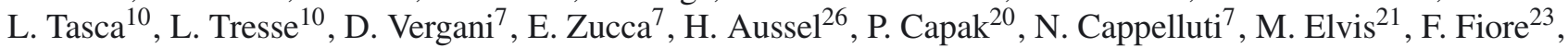 \\ G. Hasinger ${ }^{12}$, C. Impey ${ }^{22}$, E. Le Floc'h ${ }^{27}$, N. Scoville ${ }^{20}$, Y. Taniguchi ${ }^{24}$, and J. Trump ${ }^{25}$
}

(Affiliations can be found after the references)

Received 14 May 2011 / Accepted 14 September 2011

\begin{abstract}
Aims. We explore the connection between black hole growth at the center of obscured quasars selected from the XMM-COSMOS survey and the physical properties of their host galaxies. We study a bolometric regime $\left(\left\langle L_{\mathrm{bol}}\right\rangle=8 \times 10^{45} \mathrm{erg} \mathrm{s}^{-1}\right)$ where several theoretical models invoke major galaxy mergers as the main fueling channel for black hole accretion.

Methods. To derive robust estimates of the host galaxy properties, we use an SED fitting technique to distinguish the AGN and host galaxy emission. We evaluate the effect on galaxy properties estimates of being unable to remove the nuclear emission from the SED. The superb multiwavelength coverage of the COSMOS field allows us to obtain reliable estimates of the total stellar masses and star formation rates (SFRs) of the hosts. We supplement this information with a morphological analysis of the ACS/HST images, optical spectroscopy, and an X-ray spectral analysis.

Results. We confirm that obscured quasars mainly reside in massive galaxies $\left(M_{\star}>10^{10} M_{\odot}\right)$ and that the fraction of galaxies hosting such powerful quasars monotonically increases with the stellar mass. We stress the limitation of the use of rest-frame color-magnitude diagrams as a diagnostic tool for studying galaxy evolution and inferring the influence that AGN activity can have on such a process. We instead use the correlation between SFR and stellar mass found for star-forming galaxies to discuss the physical properties of the hosts. We find that at $z \sim 1$, $\approx 62 \%$ of Type-2 QSOs hosts are actively forming stars and that their rates are comparable to those measured for normal star-forming galaxies. The fraction of star-forming hosts increases with redshift: $\approx 71 \%$ at $z \sim 2$, and 100\% at $z \sim 3$. We also find that the evolution from $z \sim 1$ to $z \sim 3$ of the specific SFR of the Type-2 QSO hosts is in excellent agreement with that measured for star-forming galaxies. From the morphological analysis, we conclude that most of the objects are bulge-dominated galaxies, and that only a few of them exhibit signs of recent mergers or disks. Finally, bulge-dominated galaxies tend to host Type-2 QSOs with low Eddington ratios $(\lambda<0.1)$, while disk-dominated or merging galaxies have at their centers BHs accreting at high Eddington ratios $(\lambda>0.1)$.
\end{abstract}

Key words. quasars: general - galaxies: active - galaxies: nuclei - X-rays: general - galaxies: star formation

\section{Introduction}

There is strong observational evidence that the formation and growth of supermassive black holes (SMBHs) and their host galaxies are related processes: e.g. the tight correlation between the SMBH mass and the luminosity (Kormendy \& Richstone 1995; McLure \& Dunlop 2002; Marconi \& Hunt 2003), mass (Magorrian et al. 1998), velocity dispersion (Ferrarese \& Merritt 2000; Gebhardt et al. 2000; Merritt \& Ferrarese 2001; Tremaine et al. 2002), or the structure (Graham et al. 2001) of the bulge $^{1}$. Additional evidence of the connection between the two phenomena is provided by the similarity between the global star-formation rate (SFR) and active galactic nuclei (AGN) activity: both peak at $z \approx 2$ and decline rapidly at lower (e.g., Dickinson et al. 2003; Merloni et al. 2004) and higher

$\star$ Tables 1, 2 and Appendix A are available in electronic form at http://www . aanda.org

1 See Peng (2007) and Jahnke \& Macció (2011) for alternative ideas on the origin of these scaling relations. (e.g., Wilkins et al. 2008; Brusa et al. 2009a) redshifts. From the theoretical side, a number of models have been proposed to link the formation and evolution of SMBHs to the structure formation over cosmic time (e.g., Kauffmann \& Haehnelt 2000; Somerville et al. 2001; Granato et al. 2004, 2006; Monaco \& Fontanot 2005; Croton et al. 2006; Lapi et al. 2006; Hopkins et al. 2006; Cen 2011). Some of these semi-analytical models and hydrodynamical simulations (e.g., Springel et al. 2005) invoke major mergers of gas-rich galaxies as the mechanism enabling the fueling of the central SMBHs and the building of the galaxy's bulge. Alternative fueling mechanisms have been discussed in the literature, including minor-mergers (e.g., Johansson et al. 2009), bars (e.g., Jogee 2004), disk instabilities (e.g., Genzel et al. 2008), and recycled gas from dying stars (e.g., Ciotti et al. 2010). Hopkins \& Hernquist (2009) argued that such alternative processes may be enough to fuel Seyfert-like AGNs, but that extreme mechanisms such as major mergers are necessary to provide the gas supply needed for bright quasars. Further more, some observational studies indicate that there is a morphological transition in AGN hosts around $L_{\mathrm{bol}} \approx 10^{12} L_{\odot}$ between 
a mixture of disk and elliptical galaxies to exclusively bulgedominated galaxies (see Fig. 1 of Hopkins \& Hernquist 2009, and references therein). In this framework, the interest in studying the host galaxy properties of bright quasars is clear. These objects also dominate the space density of AGN at $z \approx 2$ (e.g., Ueda et al. 2003; Hasinger et al. 2005). An obvious complication in the study of the host galaxy properties of quasars is usually the emission of the central AGN which outshines the galaxy light making it extremely difficult to derive constraints on the colors, stellar populations, and morphologies of the host. The situation clearly improves if the powerful quasar is obscured by gas and dust on parsec scales: we refer to these objects as Type-2 QSOs. Although the fraction of obscured AGN is found to decrease with luminosity in several studies (X-ray: Ueda et al. 2003; Steffen et al. 2003; Hasinger 2004, 2008; Brusa et al. 2010; optical: Simpson 2005; MIR: Maiolino et al. 2007; Treister et al. 2008), a non-negligible population of obscured QSOs is still required by X-ray background synthesis models (e.g., Gilli et al. 2007). Radio-loud Type-2 QSOs have been observed for many years thanks to radio surveys (see McCarthy 1993, for a comprehensive review), while radio-quiet Type-2 QSOs were detected more recently in Chandra and XMM-Newton X-ray surveys (Dawson et al. 2001; Norman et al. 2002; Mainieri et al. 2002; Stern et al. 2002; Della Ceca et al. 2003; Fiore et al. 2003; Perola et al. 2004; Tozzi et al. 2006; Barger et al. 2005; Mateos et al. 2005; Krumpe et al. 2008; Vignali et al. 2010), optical surveys (SDSS, Zakamska et al. 2003; Reyes et al. 2008), and midIR observations (Martínez-Sansigre et al. 2006; Polletta et al. 2008; Lanzuisi et al. 2009). The aim of this paper is to extend to quasar luminosities the study of the host galaxy properties of AGN, using a large sample of X-ray selected Type-2 QSOs. In Sect. 2, we describe the selection criteria, while in Sects. 3 and 4 we present the average spectral properties of the sample in the X-ray and optical band, respectively. Section 5 describes the method used to separate the host galaxy from the AGN emission. In Sect. 6, we illustrate the derived properties for the host galaxies, and in Sect. 7 discuss their morphological appearance. Finally, in Sect. 8 we summarize the results of this work. We quote in this paper magnitudes in the $\mathrm{AB}$ system, we use a Chabrier initial mass function (Chabrier 2003), and we assume a cosmology with $H_{0}=70 \mathrm{~km} \mathrm{~s}^{-1} \mathrm{Mpc}^{-1}, \Omega_{\mathrm{M}}=0.3$, and $\Omega_{\Lambda}=0.7$.

\section{Sample selection}

We started from the sample of $\sim 1800$ point-like X-ray sources detected in the XMM-COSMOS survey (Cappelluti et al. 2009) for which optical identifications, multiwavelength photometry, and a compilation of available redshifts from ongoing spectroscopic campaigns in the COSMOS field (Lilly et al. 2009; Trump et al. 2009) were presented in Brusa et al. (2010). For the remaining sources very accurate photometric redshifts are available (Salvato et al. 2009). For each X-ray source, we performed a detailed X-ray spectral analysis to quantify their X-ray luminosity and obscuration. The procedure adopted to extract source and background spectra is equivalent to that described by Mainieri et al. (2007). We used XSPEC ${ }^{2}$ (v11.3.2) to perform our spectral fitting analysis. We first fitted the EPIC-pn data with two basic input models: a single powerlaw $(P L)$ and a powerlaw modified by intrinsic absorption at the redshift of the source $(A P L)$. The PL model consists of two XSPEC components wabs*zpowerlw, while the APL model consists of the combination of three

\footnotetext{
${ }^{2}$ http://heasarc.gsfc.nasa.gov/docs/xanadu/xspec/
}

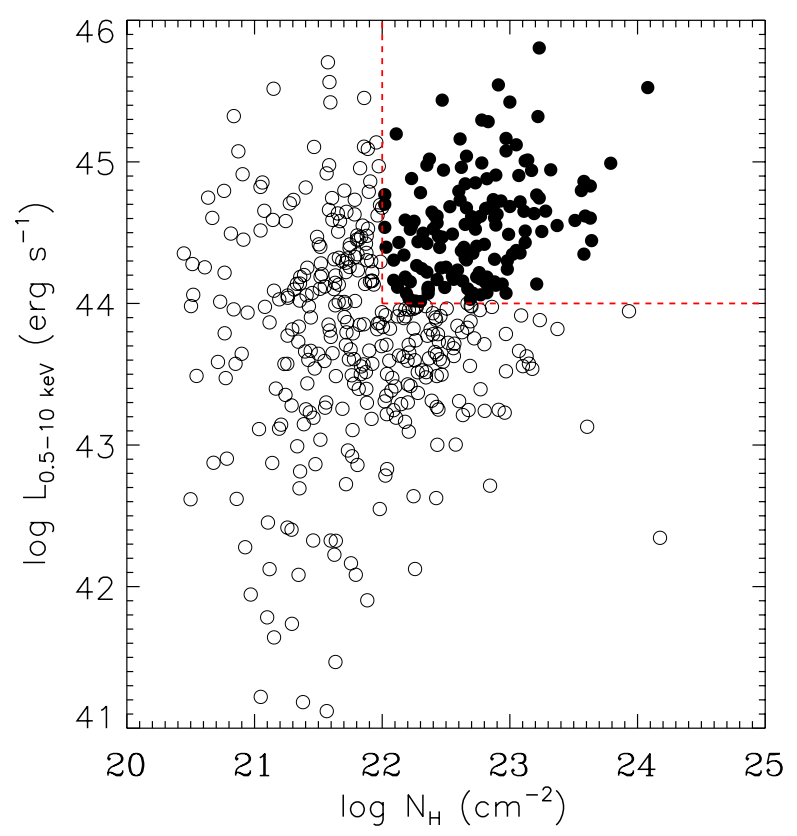

Fig. 1. X-ray luminosity in the $[0.5-10 \mathrm{keV}]$ band corrected for obscuration plotted against the $N_{\mathrm{H}}$ column density for the X-ray sources in the XMM-COSMOS survey with a redshift. The 142 Type-2 QSOs studied in this paper are indicated by filled circles. The dashed lines delimit the area where $L_{[0.5-10 \mathrm{keV}]}>10^{44} \mathrm{erg} \mathrm{s}^{-1}$ and $N_{\mathrm{H}}>10^{22} \mathrm{~cm}^{-2}$.

different components wabs*zwabs*zpowerlw. In both models, the wabs component accounts for the photoelectric absorption due to the Galactic column density and is fixed to the average value in the COSMOS region $\left(N_{\mathrm{H}}^{\mathrm{Gal}} \sim 2.7 \times 10^{20} \mathrm{~cm}^{-2}\right.$, Dickey \& Lockman 1990$)^{3}$. The zwabs component describes the photoelectric absorption using Wisconsin cross-sections (Morrison \& McCammon 1983) and the only two parameters are the equivalent hydrogen column density and the source redshift. zpowerlw is a single power-law parametrized by the photon index, the redshift, and a normalization factor ${ }^{4}$. Additional components are included to describe the presence of soft-excess or the Fe K $\alpha$ emission line. The model fits yield the power-law photon index $\Gamma$, and the X-ray luminosity in both the [0.5-2] and [2-10] keV restframe bands, and the APL model also provides the intrinsic column density $N_{\mathrm{H}}$.

We used the following selection criteria to select Type-2 QSOs: a) de-absorbed $L_{\mathrm{X}}[0.5-10 \mathrm{keV}]>10^{44} \mathrm{erg} \mathrm{s}^{-1}$; b) $N_{\mathrm{H}}>$ $10^{22} \mathrm{~cm}^{-2}$ (see also Mainieri et al. 2002; Szokoly et al. 2004; Tozzi et al. 2006; Krumpe et al. 2008). We excluded four sources (XID $=82,215,2618,5208$ ) that would formally match these criteria but for which Brusa et al. (2010) proved that the XMM-Newton emission is the blend of two Chandra sources. The final sample of X-ray selected Type-2 QSOs consists of 142 objects $^{5}$.

In Fig. 1, these candidates are plotted in the X-ray luminosity versus column density plane. The redshift distribution of the Type-2 QSO sample is shown in Fig. 2: $33(\approx 23 \%)$ of them have

3 This is an average value for the Galactic $N_{\mathrm{H}}$ in the COSMOS area, where $N_{\mathrm{H}}^{\mathrm{Gal}}$ is in the range [2.5-2.9] $\times 10^{20} \mathrm{~cm}^{-2}$. This range in Galactic column density does not affect the results of our spectral analysis.

${ }^{4}$ We refer the reader to http://heasarc.gsfc.nasa.gov/docs/ xanadu/xspec/ for further details on the spectral models.

5 Source XID-2028 discussed in Brusa et al. (2010) is not included in this paper because a re-analysis of the $\mathrm{X}$-ray spectrum returned a column density value of $\log \left(N_{\mathrm{H}}\right)=21.7 \pm 0.1 \mathrm{~cm}^{-2}$. 


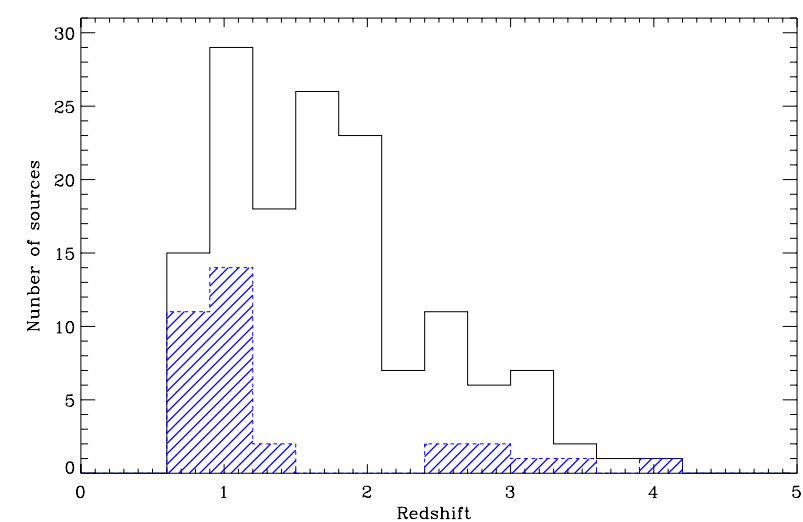

Fig. 2. Redshift distribution of the Type-2 QSO sample. The hatched histogram is for spectroscopic redshifts, while the empty one includes both spectroscopic and photometric redshifts.

a spectroscopic redshift. For this sub-sample of sources, we verified that no broad lines $\left(F W H M>2000 \mathrm{~km} \mathrm{~s}^{-1}\right)$ are present in the optical spectrum. For the remaining $77 \%$ of the sample, we used the very accurate photometric redshifts (Salvato et al. 2009). The $\mathrm{X}$-ray selected sample of Type-2 QSOs presented in this work is complementary to the SDSS optically selected one presented by Zakamska et al. (2003) and Reyes et al. (2008) in terms of redshift range covered: the SDSS sample based on [OIII] luminosities cannot be extended to redshifts higher than $z \approx 0.8$, while our sample is almost entirely at $z>0.8$.

From the absorption-corrected luminosities in the rest-frame [2-10 keV] energy band, we estimated the bolometric luminosities for the Type-2 QSOs using the luminosity-dependent bolometric correction factor $\left(k_{2-10 \mathrm{keV}}\right)$ of Marconi et al. (2004). For the X-ray luminosities sampled by our Type-2 QSOs, $k_{2-10 \mathrm{keV}}$ is in the range $[25,120]$, and the bolometric luminosities cover the range $L_{\text {bol }}=\left[1.2 \times 10^{45}, 4.5 \times 10^{47}\right] \mathrm{erg} \mathrm{s}^{-1}$ with a mean value of $\left\langle L_{\mathrm{bol}}\right\rangle \approx 8 \times 10^{45} \mathrm{erg} \mathrm{s}^{-1}$. A limitation of this approach is the intrinsic spread in AGN SEDs, which could lead to significant differences in the bolometric correction from that predicted based on a mean energy distribution for AGN (see Vasudevan \& Fabian 2007; Lusso et al. 2010). From these bolometric luminosities, and assuming a standard radiative efficiency of $\epsilon_{\mathrm{rad}}=0.1$, we derive a median accretion rate onto the black holes, $\dot{M}=L_{\text {bol }} / \epsilon_{\text {rad }} c^{2}$, of approximately $\approx 1 M_{\odot} /$ year, similar to what observed for optically selected Type-1 QSOs (e.g., Kollmeier et al. 2006; Hopkins et al. 2009).

\section{Stacked X-ray spectrum}

In order to study the average X-ray properties of our Type-2 QSO sample, we obtain a stacked X-ray spectrum using the EPIC-pn data. To avoid the possibility of the blurring and broadening of the $\mathrm{Fe} \mathrm{K} \alpha$ line caused by the uncertainties in the photometric redshifts, we first considered only the 33 sources with a spectroscopic redshift. The background-subtracted data of individual objects in the energy range corresponding to the restframe $2-10 \mathrm{keV}$ were divided into 40 intervals so that each interval corresponds to a rest-frame $200 \mathrm{eV}$. These spectra were corrected for the detector response and summed together. The details of the procedure will be presented in a future paper (Iwasawa et al., in prep.). The smallest net count recorded in the rest-frame $2-10 \mathrm{keV}$ for the spectroscopic sample is 28 photons. The resulting stacked spectrum (2682 net counts) is shown in the left panel of Fig. 3. It was further rebinned for display
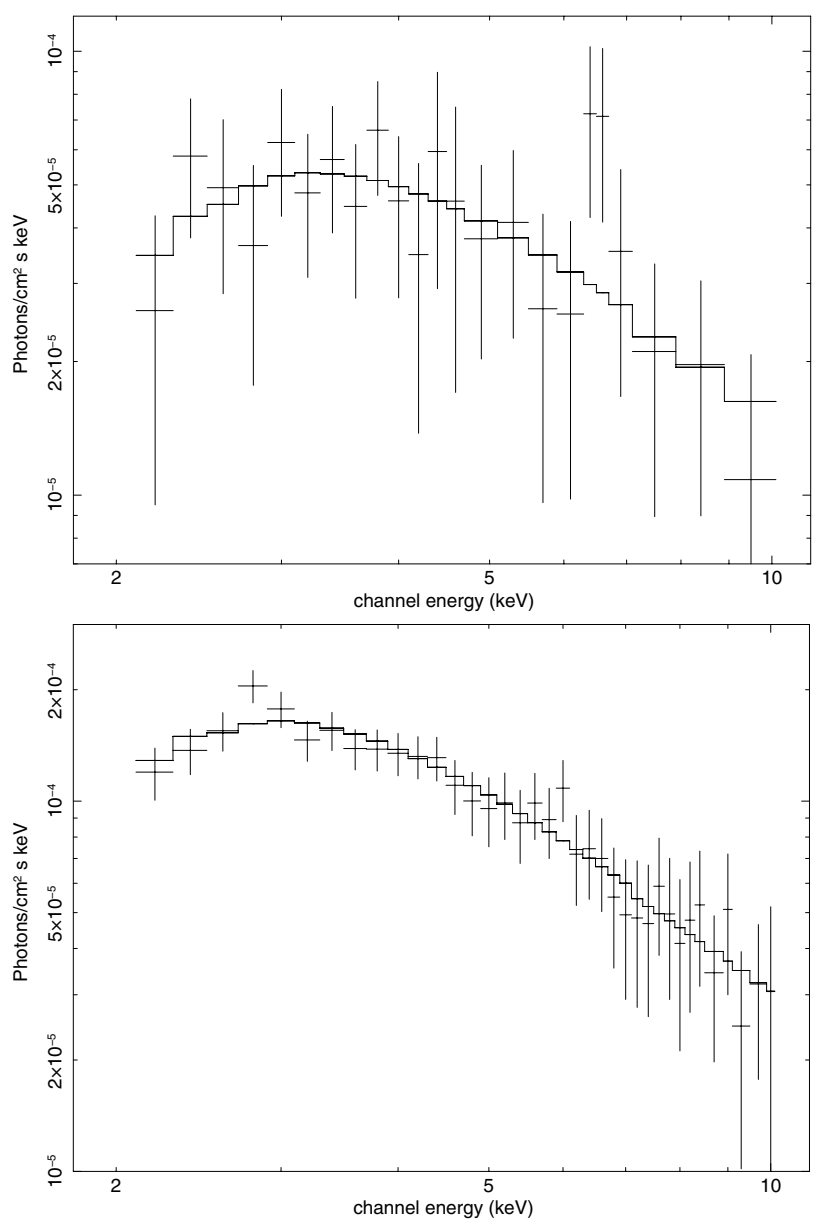

Fig. 3. Stacked X-ray spectrum of the 33 Type-2 QSOs with a spectroscopic redshift (left) and 79 Type-2 QSOs with only a photometric redshift (right). Adjacent bins were combined until they had a significant detection at least as large as $1.5 \sigma$. The solid line is the best fit to the continuum with a wabs*zwabs*powerlaw XSPEC model. In the left panel, clear residuals are present around the rest-frame $6.4 \mathrm{keV}$ energy.

purposes: adjacent bins were combined until they had a significant detection at least as large as $1.5 \sigma$. From the stacked spectrum, we measured a column density $N_{\mathrm{H}}=5.6_{3.3}^{8.3} \times 10^{22} \mathrm{~cm}^{-2}$ (quoted errors are $1 \sigma$ ), which is consistent with the average value of $\left\langle N_{\mathrm{H}}\right\rangle=5.4 \times 10^{22} \mathrm{~cm}^{-2}$ obtained from the fits to the single X-ray spectra. As is clear from the left panel of Fig. 3, an absorbed power-law model leaves large residuals around the expected energy of the $\mathrm{Fe} \mathrm{K} \alpha$ line. If we add a gaussian model centered on $6.4 \mathrm{keV}$ in the XSPEC fitting procedure, the quality of the fit improves significantly with $99 \%$ confidence according to an F-test. For the $\mathrm{Fe} \mathrm{K} \alpha$ equivalent width, we measured a value of $E W=104 \mathrm{eV}$. Taking into account the known anti-correlation between the $\mathrm{Fe} \mathrm{K} \alpha$ and the X-ray luminosity (Iwasawa \& Taniguchi 1993), this value is consistent with typical $E W$ values for Compton-thin AGNs (Bianchi et al. 2007; Chaudhary et al. 2010).

We repeated this procedure for the photometric redshift sample selecting objects that have more than 30 counts in the rest-frame $2-10 \mathrm{keV}$ band. This selection leaves $79 \mathrm{ob}-$ jects out of 112 and the corresponding stacked X-ray spectrum (6005 net counts) is shown in the right panel of Fig. 3. Owing to the larger number of objects, the final spectrum has more counts and consequently the uncertainties in the column density values are smaller, $N_{\mathrm{H}}=4.7_{3.9}^{5.5} \times 10^{22} \mathrm{~cm}^{-2}$. At the same time, the 


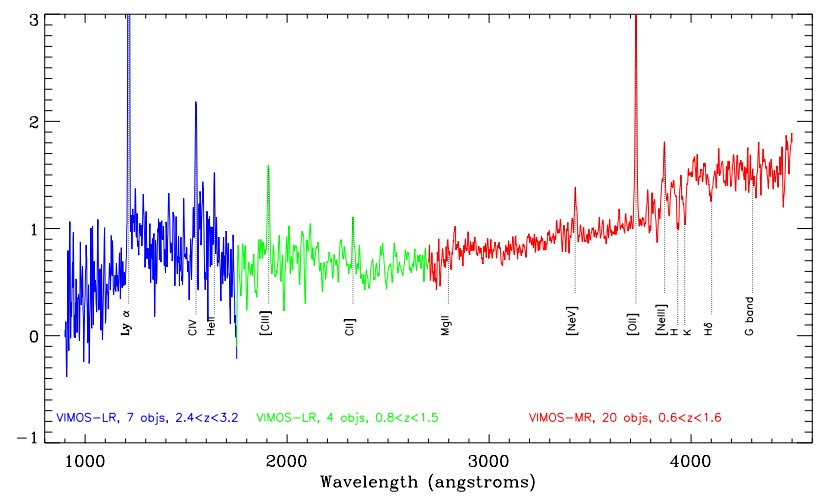

Fig. 4. Stacked optical spectrum of 31 Type-2 QSOs from the zCOSMOS survey. The LR and MR spectra have been grouped in redshift bins, as specified in the legend inside the figure, in order to have a common wavelength coverage between them. The more evident emission/absorption lines are labeled in the figure.

significance of $\mathrm{Fe} \mathrm{K} \alpha$ is significantly lower, and we ascribed this to the uncertainties, although small, in the photometric redshifts that tend to blur out the signal. In fact, an uncertainty in the photometric redshift value as low as $\Delta_{z}=0.02 \times(1+z)$ would move the centroid of the $6.4 \mathrm{keV}$ line outside the rest-frame energy bin of $200 \mathrm{eV}$ used in Fig. 3.

\section{Optical spectroscopy}

Of the 33 Type-2 QSOs with a spectroscopic redshift, 31 have an optical spectrum from the zCOSMOS survey, while the remaining two were observed with the IMACS spectrograph at Magellan. We used the 31 zCOSMOS spectra to construct an average optical spectrum for the Type-2 QSO sample. We grouped the spectra into redshift bins to ensure that each bin had a common spectral range. The VIMOS low-resolution (LR) spectra obtained with the LR-blue grism (3600-6800 ̊) were divided into two redshift bins: seven objects in $2.4<z<3.2$ and four objects in $0.8<z<1.5$. An additional twenty medium-resolution (MR) spectra (5550-9650 $\AA$ ) covering the redshift range $0.6<z<1.6$ were stacked together. The stacked optical spectrum covers the rest-frame wavelength range from 900 to $4500 \AA$ (see Fig. 4).

Both $[\mathrm{NeV}] 3426$ and $[\mathrm{OII}] 3727$ are clearly detected, and the ratio of the two lines is $[\mathrm{OII}] /[\mathrm{NeV}] \sim 4.1$. A similar value for the lines ratio was measured for a [OIII]5007 selected sample of SDSS Type-2 QSOs by Zakamska et al. (2003). In addition, Gilli et al. (2010) found a ratio $[\mathrm{OII}] /[\mathrm{NeV}]>4$ for most of the obscured quasars selected from the SDSS using the ratio of the 2-10 keV flux to the [NeV]3426 emission line flux.

We considered two features in the stacked optical spectrum of Fig. 4: the $4000 \AA$ break, $D_{n}(4000)$, and the $\mathrm{H} \delta$ absorption line. Because of the wavelengths of these two features, the following discussion is limited to the 20 Type-2 QSOs in the redshift range $0.6<z<1.6$ with a VIMOS-MR spectrum, hence the mean optical spectra may not be representative of the overall population of Type-2 QSOs. The $D_{n}(4000)$ is a rough stellar age indicator, with small values of the break for young stellar populations and large values of the index for old stellar populations. Following the definition of Balogh et al. (1999), we derived the $D_{n}(4000)$ as the ratio of the fluxes measured in the [3850-3950] $\AA$ to [4000-4100] $\AA$ bands. In the first band, we subtracted from the stacked spectrum the emission associated with the [NeIII] line. The second age indicator is based on the $\mathrm{H} \delta$ absorption line, i.e. the $\mathrm{H} \delta_{\mathrm{A}}$ index defined by Worthey \& Ottaviani (1997): the continuum is estimated from the average flux in the two bands [4041.60, 4079.75] $\AA$ and $[4128.50,4161.00] \AA$, and the index is expressed in terms of an equivalent width integrating within the band [4083.50, 4122.25] $\AA$. A strong $\mathrm{H} \delta$ absorption line is a signature of a large fraction of young stars, hence a recent burst of star formation. We measured $D_{n}(4000)=1.19 \pm 0.02$ and $\mathrm{H} \delta_{\mathrm{A}}=4.7 \pm 0.4$. We can compare these values with Fig. 7 of Winter et al. (2010). These authors used the two spectral features as age indicators in the study of local AGN selected in the 14-195 keV band with SWIFT. Our values fall in a region where the spectral models have a significant (>30\%) contribution from a young stellar populations.

\section{Distinguishing the AGN and host galaxy emission with SED fitting}

The main goal of our study is to use the rich multiwavelength coverage of the COSMOS field to constrain the properties of the galaxies hosting Type-2 QSOs. In particular, we wish to estimate their stellar mass and SFR. The technique we used is a detailed model fitting of the total SED of the Type-2 QSOs for 14 different photometric bands that encompass optical to MIR wavelengths: six SUBARU bands $(B, V, g, r, i, z)$; $U, J$, and $K$ bands from CFHT; four Spitzer/IRAC channels, and $24 \mu \mathrm{m}$ from Spitzer/MIPS (see Brusa et al. 2010, and references therein). This allows us to sample a wide wavelength interval ranging from $\sim 3800 \AA$ ( $\left.U_{\mathrm{CFHT}}\right)$ to $24 \mu \mathrm{m}$. As already presented in Merloni et al. (2010), we fitted the observed SED with a grid of models compiled by combining AGN and host galaxy templates. For the AGN component, we adopted the Richards et al. (2006) mean QSO SED derived from the study of 259 IR selected quasars with both Sloan Digital Sky Survey and Spitzer photometry. We allow for extinction of the nuclear AGN light by applying a SMC-like dust-reddening law (Prevot et al. 1984) of the form: $A_{\lambda} / E(B-V)=1.39 \lambda_{\mu \mathrm{m}}^{-1.2}$. The $E(B-V)$ values for the AGN component are allowed to vary in the range $1 \leq E(B-V) \leq 9$. Assuming a Galactic gas-to-dust ratio, our selection criteria $N_{\mathrm{H}}>10^{22} \mathrm{~cm}^{-2}$ would correspond to $E(B-V)>1.7$, but there is evidence (e.g., Maiolino et al. $2001)$ that the $E(B-V) / N_{\mathrm{H}}$ ratio in the circumnuclear region of an AGN may be lower than the Galactic value, at least for part of the AGN population. Our choice to set the lower boundary to $E(B-V)=1$ is a compromise between allowing lower than Galactic values for the dust-to-gas ratio and avoid degeneracies in the SED fitting procedure caused by the inclusion of an unobscured AGN template. Spectropolarimetry observations of Type-2 QSOs from the SDSS (Zakamska et al. 2005) and of narrow line radio galaxies (Tadhunter et al. 2002) show that in some sources there could be a non-negligible constribution from scattered quasar light. Assuming that the scattering process is independent of wavelength (Vernet et al. 2001), the SED of an unobscured quasars should be a good representation of this additional component. To quantify the effect of AGN scattered light on the estimates of the host galaxies properties presented in the next section, we repeated the SED fitting with $0 \leq E(B-V) \leq 9$ for the AGN component. The values for the host stellar mass are essentially unchanged: for $97 \%$ of the objects the difference in stellar mass is less than 5\%. Stellar population synthesis (SPS) models, as the one of Bruzual \& Charlot (2003) used in this paper, combine individual simple stellar population spectra to match the observed broad band photometry of a galaxy. Old stellar populations are the main contributor to the total galaxy stellar 
mass, and their SED dominates at wavelength above $\approx 600 \mathrm{~nm}$. This explains why adding the SED of an unobscured AGN, that would contribute significantly mostly in the UV, does not affect the stellar mass estimates based on SPS models. As for the SFR, we find that scattered AGN light would change significantly the values of $S F R$ for $\approx 15 \%$ of the objects. Therefore, in spite of the possible multi-component nature of the optical continuum, the SED fits unambiguously require the presence of a young stellar population for the majority, $\approx 85 \%$, of the objects. We also verified that the spread on SFR values, generated from the inclusion or not of an unobscured AGN component in the SED fitting procudure, does not affect the overall properties of the host galaxies population (see Figs. 8-11). As for the upper boundary in the reddening for the AGN component, $E(B-V)<9$, it corresponds to the average X-ray column density of our Type2 QSO sample of $N_{\mathrm{H}} \sim 5 \times 10^{22} \mathrm{~cm}^{-2}$ for a Galactic dust-to-gas ratio. We verified that allowing higher values of $E(B-V)$ would not affect the values for the physical quantities derived from the SED fitting, since an AGN template with $E(B-V)=9$ has practically no significant impact below $\approx 2 \mu \mathrm{m}$.

While Merloni et al. (2010), dealing with unobscured QSOs, assumed that all the $24 \mu \mathrm{m}$ flux is due to the AGN, we tried to estimate the AGN contribution at those wavelengths using the results of Gandhi et al. (2009). These authors found a strong correlation between the core luminosity at $12.3 \mu \mathrm{m}$ and the X-ray luminosity in the [2-10 keV] band for a sample of local Seyfert galaxies. In the mid-infrared, they used high-resolution observations with the VISIR/VLT instrument, and owing to the superb angular resolution of those images were able to minimize the contamination by unresolved extended emission to the core fluxes. We used their Eq. (2) for the best-fit correlation obtained by considering only the 22 well-resolved sources

$\log \left(\frac{L_{12.3 \mu \mathrm{m}}}{10^{43}}\right)=(0.19 \pm 0.05)+(1.11 \pm 0.07) \log \left(\frac{L_{2-10 \mathrm{keV}}}{10^{43}}\right)$.

Using the formula above, we estimated the AGN flux at $12.3 \mu \mathrm{m}$ from the $L[2-10 \mathrm{keV}]$ luminosities corrected for obscuration obtained from the X-ray spectral analysis. We associated an error in this mid-infrared flux using the dispersion observed by Gandhi et al. (2009) in the ratio of mid-infrared to X-ray luminosity, $\sigma\left(\log L_{\mathrm{MIR}} / L_{\mathrm{X}}\right)=0.18$ (see their Table 2 and Fig. 2). Finally, we included in the SED fitting procedure the constraint that the AGN component matches this predicted flux in the mid-infrared.

For the host galaxy, we created a library of synthetic spectra using the well-known stellar population synthesis models of Bruzual \& Charlot (2003). We compiled ten exponentially declining star-formation histories (SFH) $S F R \propto \mathrm{e}^{-t_{\text {age }} / \tau}$ with e-folding times $(\tau)$ ranging from 0.1 to $30 \mathrm{Gyr}$, plus a model with constant star formation. For each of these SFH, we calculated the synthetic spectrum at different ages, $t_{\text {age }}$, ranging from 50 Myr to $5 \mathrm{Gyr}$, subject only to the constraint that the age should be younger than the age of the Universe at the redshift of the source. Finally, we included dust extinction, modeled using the Calzetti's law (Calzetti et al. 2000), with values in the range $0 \leq E(B-V) \leq 0.5$. Following Fontana et al. (2004) and Pozzetti et al. (2007), we imposed the prior $E(B-V)<0.15$ if $t_{\text {age }} / \tau>4$, a significant extinction being allowed only for galaxies with a high SFR. We adopted a Chabrier (2003) initial mass function (IMF) ${ }^{6}$.

\footnotetext{
${ }^{6}$ Different choices of IMF lead to systematic shifts in the estimated stellar masses for any given SED, with the maximum shift for a Salpeter IMF (Salpeter 1955 ) given by $M_{\star, \text { Salpeter }} \approx M_{\star, \text { Chabrier }} \times 1.7$ (see e.g., Pozzetti et al. 2007; Ilbert et al. 2010).
}
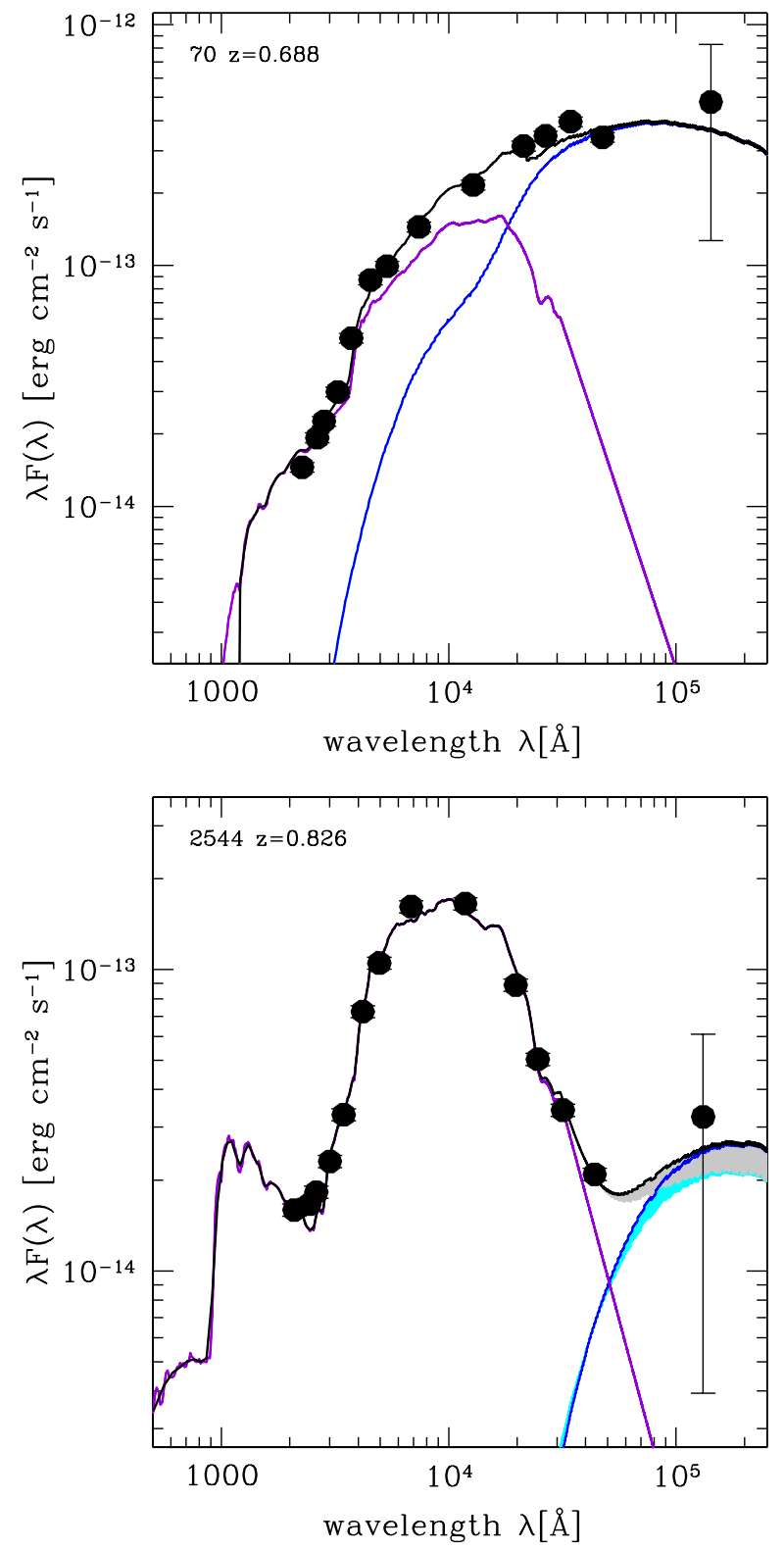

Fig. 5. Two examples of SED decomposition. Black circles are the observed photometry in the rest-frame. Purple and blue lines correspond respectively to the galaxy and AGN template found as the best fit solution, while the black line shows their sum.

Finally, an additional component that could contribute to the UV continuum of the SED is nebular continuum (see Dickson et al. 1995). For a sample of ten narrow line radio galaxies, possibly radio-loud Type-2 QSOs, Tadhunter et al. (2002) calculated the fractional contribution of this componenent to the UV flux in the range $3-30 \%$. In this work, we will not consider the possible contribution of the nebular continuum. We note that at least for stellar mass estimates the effect of this component should be negligible: the intensity of the nebular continuum drops quickly with wavelength and becomes extremelly faint at $\lambda>500 \mathrm{~nm}$, where we expect to be more sensitive to the galaxy stellar mass.

For each one of the Type-2 QSOs, we derived the best fit SED from the minimization of the $\chi^{2}$ comparing the observed and template fluxes at the redshift of the source. The combination of templates allowed us to disentangle the emission of the host galaxy from the contribution of the central black hole. We show in Fig. 5 two examples of this composite fit. From the best 

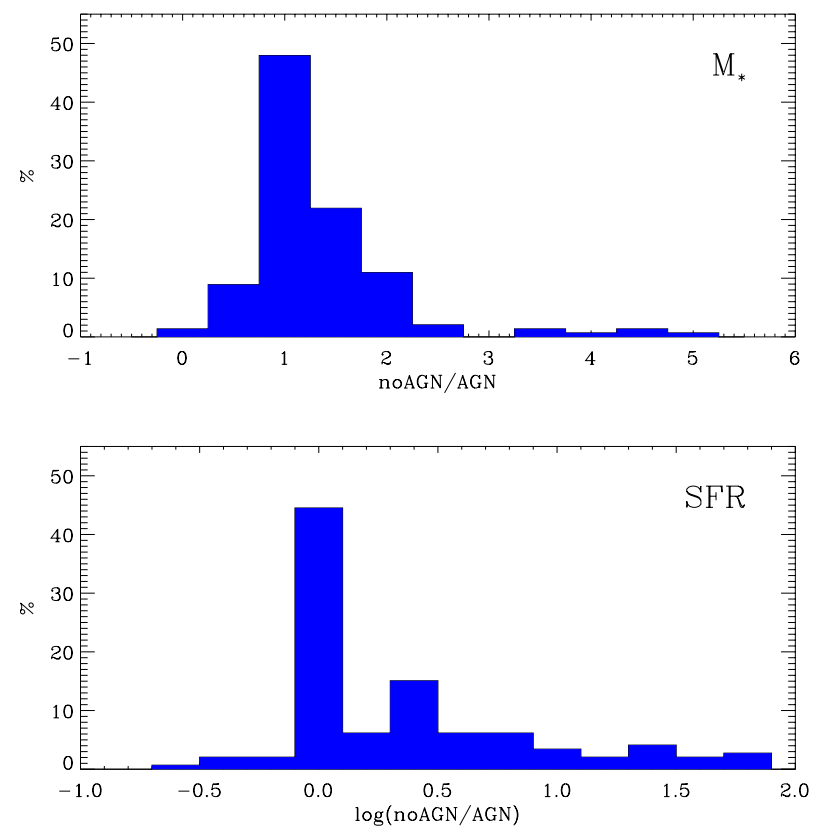

Fig. 6. Comparison between the estimates of $M_{\star}$ (top panel) and SFR (bottom panel) obtained with the SED fitting technique using only galaxies templates $(n o A G N)$ or a combination of galaxies and AGN templates $(A G N)$.

fit SED, we used the galaxy component to estimate both the stellar mass and SFR of the host. For each one of these physical quantities, we estimated the confidence region corresponding to $68 \%$ of confidence level for one interesting parameter, imposing that $\chi^{2}-\chi_{\text {BEST-FIT }}^{2} \leq 1$ (Avni 1976, and Table 1 therein). The best-fit values and errors obtained are reported in Table 1. We flagged in the same table the sources without a reliable fit to their SED for various reasons (e.g. photometry contaminated by a nearby bright object).

\section{Host galaxy properties}

\subsection{Stellar mass}

In this section we estimate the stellar masses of the Type2 QSOs host galaxies. Several previous studies of Type2 AGN host galaxy properties have assumed that the contamination of the AGN continuum to their measurements is negligible (e.g., Silverman et al. 2008, 2009; Schawinski et al. 2010; Xue et al. 2010). However, those studies were focused on moderate luminosity AGNs, e.g. $\log \left(L_{\mathrm{X}}<44\right) \mathrm{erg} \mathrm{s}^{-1}$. The AGN sample presented in this work consists of obscured nuclei, but they are all intrinsically powerful quasars, hence may still contribute in a non-negligible way to the overall photometry. It is then important to model this contamination, which would otherwise bias the measurement of the stellar mass from the SED fitting. To quantify the effect of the nuclear component, we performed the SED fitting using either the combination of galaxy and AGN templates described in the previous section as well as only galaxy templates. In the top panel of Fig. 6, we compared the stellar mass estimates obtained with the two sets of templates. While for the majority of the objects, the introduction of an AGN component in the SED fit is irrelevant, there are a $10 \%$ of cases where neglecting these components would cause more than a factor of two overestimation of the stellar masses.

From the stellar masses reported in Table 1, one can immediately see that the hosts of Type-2 QSOs are mainly massive

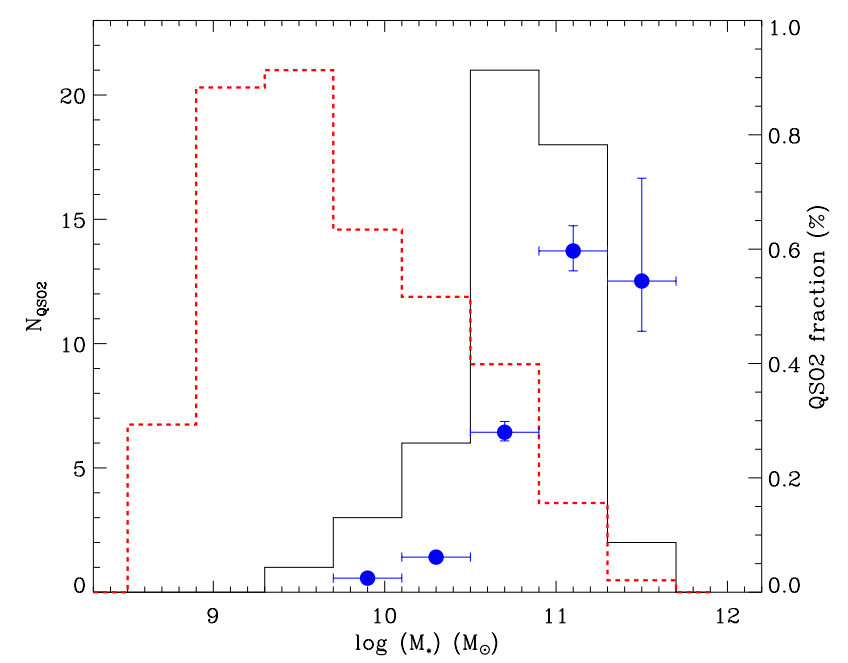

Fig. 7. Stellar mass distribution of the Type-2 QSOs host galaxies (solid histogram) and of the galaxies parent sample (dashed histogram) with $0.8<z<1.5$. The second histogram has been rescaled for graphical reasons by a factor $\sim 1000$. The data points represent the fraction of galaxies hosting Type-2 QSOs in bin of stellar masses (see Sect. 6.1).

galaxies. To quantify this impression, we need to compare the stellar mass distribution of these hosts with a representative sample of galaxies that matches their properties. We selected a parent sample of galaxies detected in the $3.6 \mu \mathrm{m}$ IRAC channel from the COSMOS survey using the stellar masses measured by Ilbert et al. (2010). We considered only the redshift range $z=[0.8,1.5]$, where a high completeness in stellar mass above $\approx 5 \times 10^{9} M_{\odot}$ and accurate photometric redshifts are ensured (see Fig. 8 of Ilbert et al. 2010). The final parent sample includes 76061 galaxies. The sensitivity limits of the $X M M-N e w t o n$ observations vary spatially across the field (see Fig. 5 of Cappelluti et al. 2009), hence to derive the fraction of galaxies hosting a Type-2 QSO as a function of the stellar mass, we followed the technique discussed in Sect. 3.1 of Lehmer et al. (2007; see also Silverman et al. 2008, 2009). The Type-2 QSOs fraction for each stellar mass bin is determined by summing over the sample of Type-2 QSOs $\left(N_{\mathrm{QSO} 2}\right)$

$f=\sum_{i=1}^{N_{\mathrm{QSO} 2}} \frac{1}{N_{\mathrm{gal}, i}}$,

where $N_{\mathrm{gal}, i}$ represents the number of galaxies for which we might have detected an AGN with X-ray luminosity $L_{X, i}$. We have plotted in Fig. 7 the fraction of galaxies hosting a Type2 QSOs. This fraction monotonically increases with stellar masses going from $0.02 \%$ at $M_{\star} \approx 8 \times 10^{9} M_{\odot}$ to $\approx 0.6 \%$ at $M_{\star} \approx 10^{11} M_{\odot}$.

This trend is consistent to what has been found for the overall AGN population (Kauffmann et al. 2003; Best et al. 2005; Silverman et al. 2009; Brusa et al. 2009a; Xue et al. 2010).

For our sample of Type-2 QSOs, we do not detect broad lines and therefore we cannot derive "virial" black hole masses using the calibration obtained with reverberation mapping on local AGN (e.g., Kaspi et al. 2000). To roughly estimate the mass of the black holes powering these obscured quasars, we used the local $M_{\mathrm{BH}}-M_{\star}$ scaling relation provided by Häring \& Rix (2004). Since this relation was derived locally, we took into account a possible redshift evolution parametrized by Merloni et al. (2010): $\Delta \log \left(M_{\mathrm{BH}} / M_{\star}\right)(z)=\delta_{2} \log (1+z)$, where $\delta_{2}=0.68 \pm 0.12$. We found that most $(\sim 90 \%)$ of the 


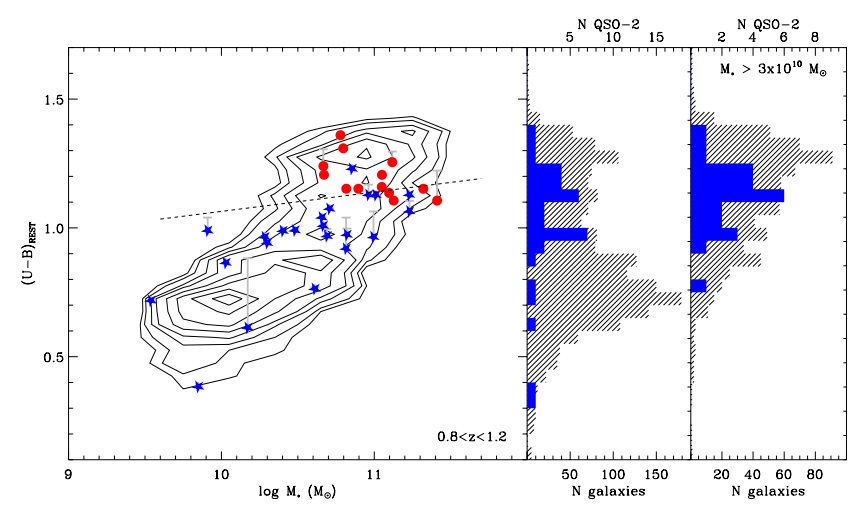

Fig. 8. Left panel. Color-mass diagram in the redshift range $0.8<z<$ 1.2 for the comparison sample of normal galaxies (gray contours), and the Type-2 QSOs hosts. The rest-frame colors of the Type-2 QSOs hosts have been measured after removing the contribution from the AGN. Star-forming galaxies are indicated with the star symbol, while the circles correspond to passive galaxies. The dashed curve is the dividing line between red passive and blue star-forming galaxies at $z=1 \mathrm{ac}-$ cording to Peng et al. (2010). The vertical bars show the variation in rest-frame colors if an unobscured AGN component is included in the SED fitting procedure (see Sect. 5). Central panel. $U-B$ rest-frame color distribution for the Type-2 QSOs host galaxies (filled histogram) and the comparison sample of normal galaxies (hatched histogram) in the redshift range $0.8<z<1.2$. Right panel. As in the central panel but only for sources with $M_{\star}>3 \times 10^{10} M_{\odot}$.

Type-2 QSOs have black hole masses in the range $\log \left(M_{\mathrm{BH}}\right)=$ [7.5, 9.5] $M_{\odot}$. Using these estimates of black hole masses and the bolometric luminosities derived at the end of Sect. 2, we computed the Eddington ratio $\left(\lambda_{\text {Edd }}=L_{\text {bol }} / L_{\text {Edd }}\right.$, where $L_{\text {Edd }}=$ $\left.1.38 \times 10^{38} M_{\mathrm{BH}} / M_{\odot}\right)$. We found that $\approx 50 \%$ of the Type-2 QSOs have $\lambda_{\text {Edd }}>0.1$, the remaining half being in the range $0.01<\lambda_{\text {Edd }}<0.1$.

\subsection{Rest-frame colors}

Galaxies display a color bimodality in both the local Universe (Strateva et al. 2001; Hogg et al. 2002; Blanton et al. 2003) and at higher redshift (Bell et al. 2004; Weiner et al. 2005, up to $z \approx 1$; Franzetti et al. 2007; Cirasuolo et al. 2007, up to $z \approx 1.5$; Giallongo et al. 2005; Cassata et al. 2008; Williams et al. 2009, up to $z \approx 2$ ). This bimodality in red-sequence and blue-cloud galaxies has been interpreted as evidence of a dichotomy in the star-formation and merging histories of galaxies (e.g., Menci et al. 2005). Color-magnitude diagrams have been used as tools in galaxy evolution studies, and since many models invoke AGN feedback as an important player in this evolution, it may be interesting to locate the hosts of Type- 2 quasars in those diagrams.

From the galaxy component of the best fit to the Type2 QSO SED (see Sect. 5), we derived rest-frame colors for the hosts in the Johnson-Kron-Cousins system. The inclusion of an AGN component in our SED fitting procedure prevents contamination from the nuclear emission to the colors of the host galaxy ${ }^{7}$.

From the histogram in the central panel of Fig. 8, one could conclude that the host galaxies of Type-2 QSOs mainly have rest-frame colors in-between the blue and red galaxy populations, similarly to what has already been reported for

\footnotetext{
7 See the discussion in Appendix A of Hickox et al. (2009) about the contribution of the nucleus to optical colors of X-ray and radio-selected AGNs if the SED fitting is done using only galaxy templates.
}

lower luminosity X-ray/optically selected AGNs (Nandra et al. 2007; Martin et al. 2007; Rovilos \& Georgantoupoulos 2007; Silverman et al. 2008; Georgakakis et al. 2008; Schawinski et al. 2009; Hickox et al. 2009). Silverman et al. (2009) demonstrated that if one considers stellar-mass rather than luminosity selected samples of AGN and parent galaxies, the fraction of galaxies hosting an AGN does not decline towards bluer colors. Xue et al. (2010) confirmed that the AGN fraction remains nearly constant as a function of color for a stellar mass selected sample of AGN from the Chandra Deep Fields. Both these works focused on AGN with luminosities below $L_{X}<10^{44} \mathrm{erg} \mathrm{s}^{-1}$. Our sample of Type-2 QSOs is by construction brighter and therefore care is required when extrapolating these previous results. If we select only the objects with $M_{\star}>4 \times 10^{10} M_{\odot}$ for which our COSMOS sample is mostly complete (see Ilbert et al. 2010), the distribution of rest-frame colors is modified as shown in the right panel of Fig. 8. In a mass-selected sample of galaxies the distribution of rest-frame colors for active and inactive galaxies is similar. Since stellar mass is likely playing a major role in determining the evolution of galaxies, mass-selected samples are more suitable when comparing the properties of the AGN hosts with those of inactive galaxies.

If we adopt the dividing line between blue star-forming and red passive galaxies as suggested by Peng et al. (2010)

$(U-B)_{\mathrm{AB}}=1.10+0.075 \log \left(\frac{m}{10^{10} M_{\odot}}\right)-0.18 z$,

using $z=1.0$, we find that $\approx 28 \%$ of the host galaxies of Type2 QSOs in the redshift range $0.8<z<1.2$ are red and $\approx 72 \%$ are blue. If were to use the $(U-B)$ versus $M_{B}$ diagram and the Willmer et al. (2006) color division, the fraction of Type-2 QSOs hosts that are classified as red galaxies would increase to $\approx 58 \%$. We stress that the use of rest-frame colors to study the properties of AGN host galaxies is subject to a proper treatment of reddening. Objects with intermediate or red colors could actually be star-forming galaxies obscured by large amounts of dust on kpc scales (e.g., see Brammer et al. 2009; Pozzetti et al. 2010). As we describe in the next section, we were able to derive estimates of the SFR of our host galaxies from the SED fitting. We can therefore use the specific SFR $\left(S S F R=S F R / M_{\star}\right)$ to divide the sample into active and quiescent galaxies depending on whether $\log \left(S S F R / \mathrm{Gyr}^{-1}\right)$ is above or below -1, i.e., galaxies that would take less or more than 10 Gyr to double their stellar mass at the present SFR, respectively (see Pozzetti et al. 2010). We find that $\approx 20 \%$ of the red galaxies are actively forming stars, and therefore their colors are due to the presence of dust rather than an old stellar population. Our result is in agreement with previous studies. Cardamone et al. (2010), using rest-frame nearinfrared colors of lower luminosity AGN hosts to separate passive from dusty galaxies, found that $\approx 25 \%$ of the hosts in the red cloud are dusty star-forming galaxies; Brusa et al. (2009b) from the SED fitting of a sample of obscured AGN in the Chandra Deep Field South reached the conclusion that the fraction of dusty star-forming hosts was up to $\approx 50 \%$ of their sample.

\subsection{SFR}

As described in Sect. 5, we estimated the SFR values of the host galaxies using the SED fitting technique. We stress that to reliably estimate the SFR of the host galaxy it is crucial to remove the contribution of the AGN to the observed photometry. As already discussed, we achieved this by performing the SED fitting with a combination of AGN and galaxy templates. To quantify 
the effect of not modeling the nuclear emission, we compared the SFR estimates obtained only with galaxy templates and a combination of galaxies and AGN SEDs, as discussed in Sect. 6.1. We find that for $\approx 40 \%$ of the objects neglecting the AGN component will overestimate the SFR by more than a factor of two, and up to a factor of fifty for a few galaxies (see bottom panel of Fig. 6).

For comparison, we also estimated the SFR using the UV continuum luminosity. Using the prescriptions of Daddi et al. (2004) for star-forming galaxies at $1.4<z<2.5$ and assuming a Chabrier IMF, we have

$\operatorname{SFR}\left(M_{\odot} \mathrm{yr}^{-1}\right)=L_{1500 \AA}^{\mathrm{corr}} / 8.85 \times 10^{27} / 1.7$,

$L_{1500 \AA}^{\text {corr }}=L_{1500 \AA} \times 10^{0.4 \times A_{1500}}$,

where $L_{1500} \AA$ is the galaxy luminosity at $1500 \AA(\mathrm{erg} / \mathrm{s} / \mathrm{Hz})$, $L_{1500 \AA}^{\text {corr }}$ is corrected for extinction by dust, and $A_{1500} \approx 10 \times$ $E(B-V)$ assuming a Calzetti's law for the dust extinction, where we use the $E(B-V)$ values derived from the best SED fit using both galaxies and AGN templates. Considering the $\approx 60$ QSO-2 hosts that match the Daddi et al. (2004) selection criteria, we find that the median ratio $\left(S F R_{\mathrm{SED}} / S F R_{\mathrm{UV}}\right)$ is $\approx 1.1$ with a dispersion of $\approx 0.6$.

One of the main goals of this work was to compare the level of star formation in the hosts of the Type-2 QSOs with that measured for "non-active" galaxies. Surveys of the local Universe (Brinchmann et al. 2004; Salim et al. 2005) found that galaxies can be divided mainly into two populations: star-forming objects exhibiting a continuous increase in SFR with $M_{\star}$ along a tight correlation and quenched galaxies with little star formation and high stellar masses. The tight correlation between SFR and $M_{\star}$ for star-forming galaxies has been confirmed to already be in place at higher redshifts (Noeske et al. 2007a; and Elbaz et al. 2007, up to $z \approx 1$; Daddi et al. 2007; and Pannella et al. 2009 , up to $z \approx 2$ ), also when using PACS/Herschel observations (Rodighiero et al. 2010). We refer to it as the main sequence (MS) of star-forming galaxies following Noeske et al. (2007a). The slopes of the MS at different redshifts appear to be the same but the normalization has decreased by a factor $\approx 3.7$ going from $z \approx 2$ to $z \approx 1$, and an additional factor $\approx 7$ at $z \approx 0$ (Daddi et al. 2007). This decrease in the activity of star-forming galaxies of a given stellar mass reflects the decline in the global SFR density with time, and a likely cause of this is gas exhaustion (e.g., Noeske et al. 2007b).

The galaxy dichotomy found using the correlation between SFR and $M_{\star}$ is more fundamental than that based on rest-frame colors: red colors do not necessarily mean that the galaxy has stopped forming stars (see the discussion in Sect. 6.2, and the red colors of some MS galaxies at high $M_{\star}$ in Fig. 17 of Elbaz et al. 2007). Our aim is to compare the properties of the host galaxies with a parent sample of normal galaxies in the context of this correlation.

In the redshift range $0.8<z<1.2$, we have 35 Type2 QSOs; and we were able to establish a good fit to their SED for $34^{8}$ of which. We find that $\approx 62 \%$ of the Type- 2 QSO host in this redshift range are actively forming stars. In the sample of star-forming galaxies used by Elbaz et al. (2007) to derive the MS at $z \approx 1$, only "blue" galaxies were considered (see their Fig. 13). For consistency, we derived rest-frame absolute magnitudes in the standard $U$ and $B$ Johnson's filters from the galaxy

\footnotetext{
8 Source 5326 has near-IR photometry contaminated by a nearby source and will not be considered in this analysis.
}

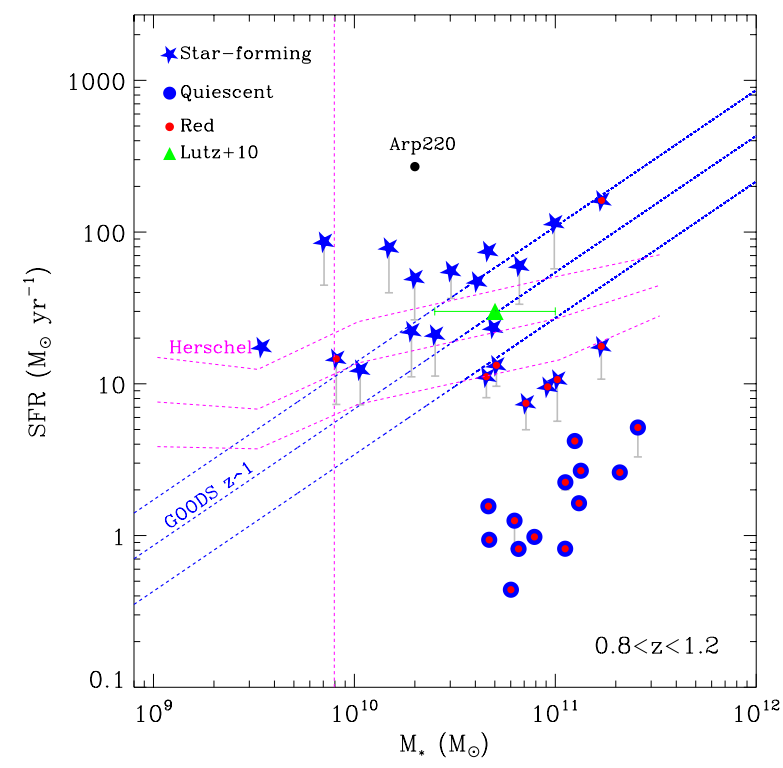

Fig. 9. SFR versus stellar masses obtained from the SED fitting for the host galaxies of the Type-2 QSOs in the redshift range $0.8<z<1.2$. Galaxies with $\log \left(\operatorname{SSFR} / \mathrm{Gyr}^{-1}\right)>-1$ are indicated with a star symbol, otherwise a circle was used. Type-2 QSO hosts with red $(U-B)$ restframe colors according to the color division by Willmer et al. (2006) have been highlighted with a smaller lighter circle. The vertical bars show the variation in SFR if an unobscured AGN component is included in the SED fitting procedure (see Sect. 5). The dashed lines are the correlation and its one- $\sigma$ confidence intervals found for blue starforming galaxies by Elbaz et al. (2007) and PACS/Herschel sources by Rodighiero et al. (2010) at $z \approx 1$. The curves have been rescaled from the original Salpeter IMF to the Chabrier IMF adopted in this work. The vertical dashed line indicates the mass completeness limit of the PACS sample. The triangle indicates the average SFR for the hosts of X-ray selected AGNs estimated by Lutz et al. (2010) by stacking $870 \mu \mathrm{m}$ LABOCA/APEX data.

component of the SED fit of our Type-2 QSO hosts, and separated them into blue and red galaxies using the color division by Willmer et al. (2006). As shown in Fig. 9, there is a mild correlation between SFR and $M_{\star}$ for the "blue" star-forming hosts. The Spearman rank correlation coefficient for these sources is $r=0.38(p=0.2)$. For the majority of our "blue" star-forming host galaxies, the level of SSFR at $z \approx 1$ is consistent with the MS of star-forming galaxies at the same redshift. In Fig. 9, we also indicated, the average SFR estimated by Lutz et al. (2010) for the hosts of X-ray selected AGN at $z \sim 1$ obtained by stacking $870 \mu \mathrm{m}$ LABOCA observations in the Extended Chandra Deep Field South. Most of these hosts have stellar masses in the range $10.4<\log \left(M_{\star}\right)<11 M_{\odot}$ (Xue et al. 2010). From this stacking analysis, the derived value of SSFR for AGN hosts is also consistent with the MS of star-forming galaxies at $z \sim 1$.

We repeated a similar analysis for the hosts in the three higher redshifts bins: $1.2<z<1.8$ (36 objects), $1.8<z<2.5$ ( 28 objects), and $2.5<z<3.2$ (12 objects). First, we find that the fraction of star-forming galaxies between our Type-2 QSO hosts increases with redshift: $66 \%, 71 \%$, and $100 \%$ respectively. We then looked at the evolution of the SSFR for Type-2 QSO hosts between redshift $z=0.8$ and $z=3$ as shown in Fig. 10 . At a first look, this figure would suggest that there is only a mild evolution, if any, of the average SSFR for Type-2 QSO hosts going from $z \sim 1$ to $z \sim 2$, while there is no evolution at all at $z>2$. The latter agrees with the constant SSFR measured from $z \sim 7$ to $z \sim 2$ for normal galaxies (e.g., Gonzalez et al. 2010), while the former disagrees with the substantial drops of 
V. Mainieri et al.: Black hole accretion and host galaxies of obscured quasars in XMM-COSMOS

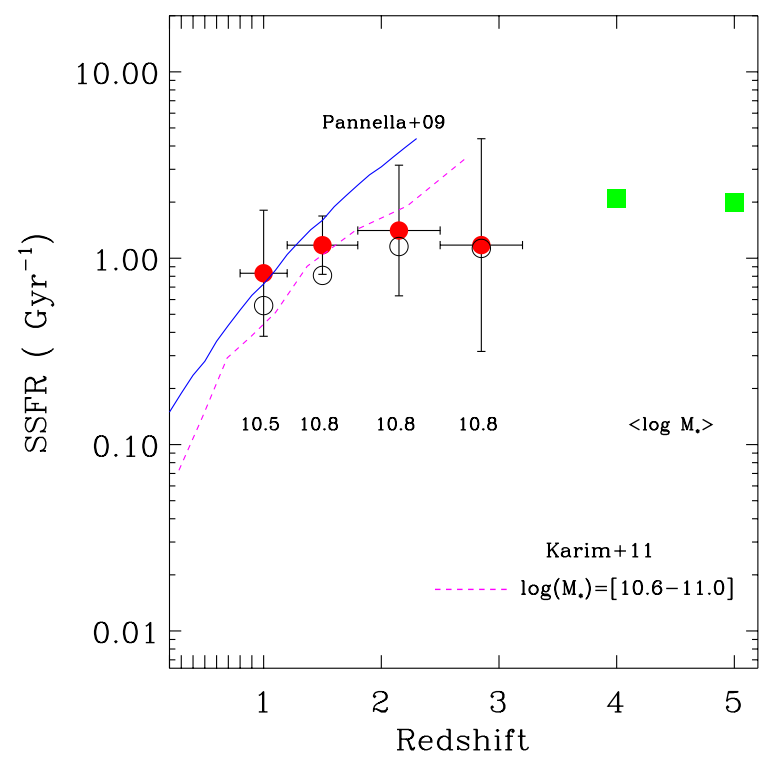

Fig. 10. Evolution of the average SSFR for Type-2 QSOs hosts as a function of redshift. For each redshift bin we report the average stellar mass of the host galaxies. Empty circles indicate the average SSFR per redshift bin if an unobscured AGN component is included in the SED fitting procedure (see Sect. 5). The continuum line reproduces the evolution law found by Pannella et al. (2009) for star-forming galaxies up to $z \sim 2$. The dashed line instead is the best fit to the SSFR redshift evolution derived by Karim et al. (2011) in the mass range $\log \left(M_{\star}\right)=$ $[10.6,11.0] M_{\odot}$. The two squares are the SSFR for normal galaxies at $z=[4,5]$ as presented by Gonzalez et al. (2010) using data from Stark et al. (2009).

the average SSFR below $z \sim 2$ (e.g., Noeske et al. 2007a; and Daddi et al. 2007). Shao et al. (2010) used Herschel/PACS observations of the GOODS-N field to estimate the SFR in X-ray selected AGNs. They find that the host far-infrared luminosity of AGN with $L_{2-10 \mathrm{keV}} \approx 10^{44} \mathrm{erg} \mathrm{s}^{-1}$ does not significantly increase from $z \sim 1$ to $z \sim 2$ (see their Fig. 4). Since the rest-frame far-infrared emission is typically dominated by the host rather than the AGN (e.g., Netzer et al. 2007) it can be used as a SFR diagnostic and therefore the Shao et al. (2010) results are consistent with ours in Fig. 10. However, submillimetre surveys of narrow line radio galaxies, possibily radio loud Type-2 QSOs, found that their FIR luminosity rises with redshift out to $z \approx 4$, and interpreted it as an increase in the star formation activity with redshift (e.g., Archibald et al. 2001; Reuland et al. 2004). We note that the samples of Type-2 QSOs in the redshift bins plotted in Fig. 10 have different mean stellar masses: at $z \sim 1$, the average stellar mass is $\left\langle M_{\star}\right\rangle \sim 3 \times 10^{10} M_{\odot}$, while it is higher, $\left\langle M_{\star}\right\rangle \sim 6 \times 10^{10} M_{\odot}$, at $z \sim 3$. Also the curve from Pannella et al. (2009) plotted in Fig. 10 is most valid for $M_{\star} \sim 3 \times 10^{10} M_{\odot}$. According to Karim et al. (2011), there is a correlation between SSFR and stellar masses, with higher mass objects having lower SSFRs. For the three redshifts bins below $z \sim 2$, we now include only the hosts with $\log \left(M_{\star}\right)=[10.6-11.0] M_{\odot}$ and consider the best fit to the redshift evolution of the SSFR for normal galaxies in the same mass range (Table 5 of Karim et al. 2011). As Fig. 11 shows, the agreement between Type-2 QSO hosts and normal galaxies is now excellent: the average SSFR for our hosts decreases by a factor $\approx 3.6$ going from $z \sim 2$ to $z \sim 1$.

\section{Morphology}

Another important diagnostic of galaxy evolution is their morphologies, which correlates with their star-formation histories.

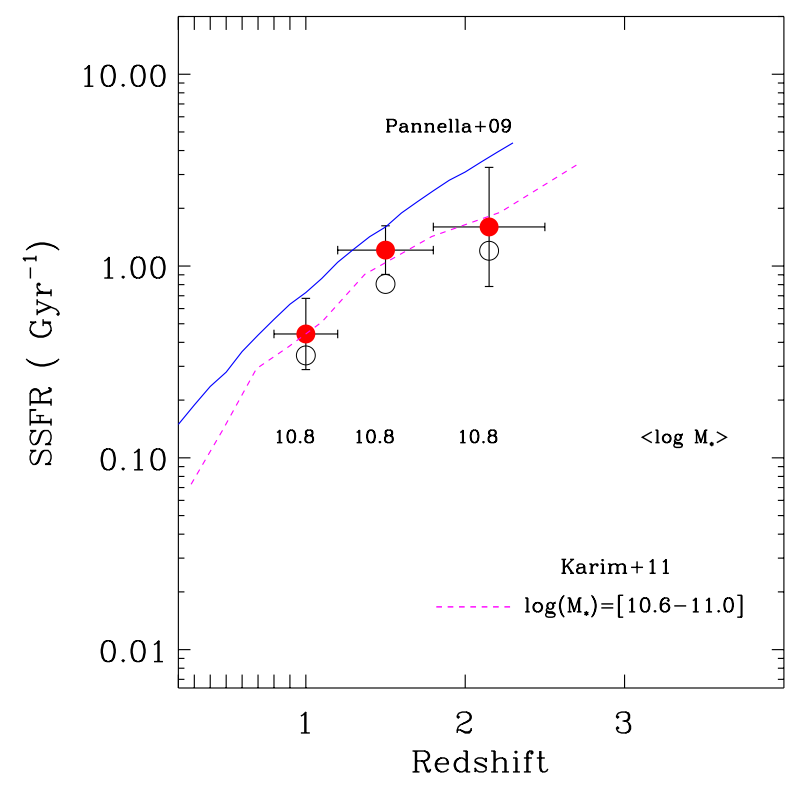

Fig. 11. Evolution of the average SSFR for Type-2 QSOs hosts with $\log \left(M_{\star}\right)=[10.6,11.0] M_{\odot}$ from $z \sim 1$ to $z \sim 2$. The lines and symbols are the same as in Fig. 10.

In Type-2 QSOs, obscuration on parsec scales prevents the light of the nuclei from outshining the host galaxy, therefore making it easier, compared to unobscured Type-1 QSOs, to study the morphological properties of their hosts. Using the Cycle 12 Hubble Space Telescope (HST) Advanced Camera for Surveys (ACS) F814W images of the COSMOS field (Scoville et al. 2007; Koekemoer et al. 2007), we studied the morphology of 34 Type-2 QSO host galaxies with $z<1.2$ and $I_{\mathrm{AB}}<24$. We used an upgraded version of the Zurich estimator of structural types (ZEST; Scarlata et al. 2007) known as ZEST+ (Carollo et al., in prep.). This new tool, based on a principal component analysis, includes additional measurements of non-parametric morphological indices for characterizing both structure and substructures. For each of the 34 host galaxies, we measured the half-light radius $\left(r_{1 / 2}\right)$, the Petrosian radius $\left(R_{\mathrm{P}}\right)$, and the ellipticity $(\epsilon)$. In addition, we derived estimates of four popular nonparametric measurements of the light distribution in galaxies (e.g., Abraham et al. 2003; Lotz et al. 2004): concentration (C), asymmetry (A), second-order moment of the brightest $20 \%$ of the galaxy flux $\left(M_{20}\right)$, and Gini coefficient $(\mathrm{G})$. The values of these parameters for each of the 34 Type-2 QSOs hosts are reported in Table 2. Using a combination of this information and after a visual inspection of the ACS images, we divided our host galaxies into three classes: bulge-dominated, disk-dominated, and possible-merger. The breakdown in morphological classes for the Type-2 QSO host galaxies at $z \leq 1.2$ is the following: $57 \%$ bulge-dominated, $23 \%$ disk-dominated, and $20 \%$ possible mergers. Color cutouts of the hosts divided by morphological classes are shown in Figs. 12-14. In Fig. 15, we plot the distribution of our host galaxies in three planes: G vs. $M_{20}$, C vs. $M_{20}$, and C vs. A. Scarlata et al. (2007) obtained reliable morphological classifications for $\sim 56000 I_{\mathrm{AB}} \leq 24$ galaxies in the COSMOS field using ZEST. For comparison, we added in Fig. 15 the contours enclosing 99\% of the Scarlata et al. (2007) galaxies belonging to the ZEST morphological classes $T=1$ (red), $T=2.0$ or 2.1 (blue), which correspond to disk galaxies with a Sersic index $n>1.25$, and $T=3$ (green). While in the first two panels our morphological classification agrees quite well with the large galaxies sample studied 


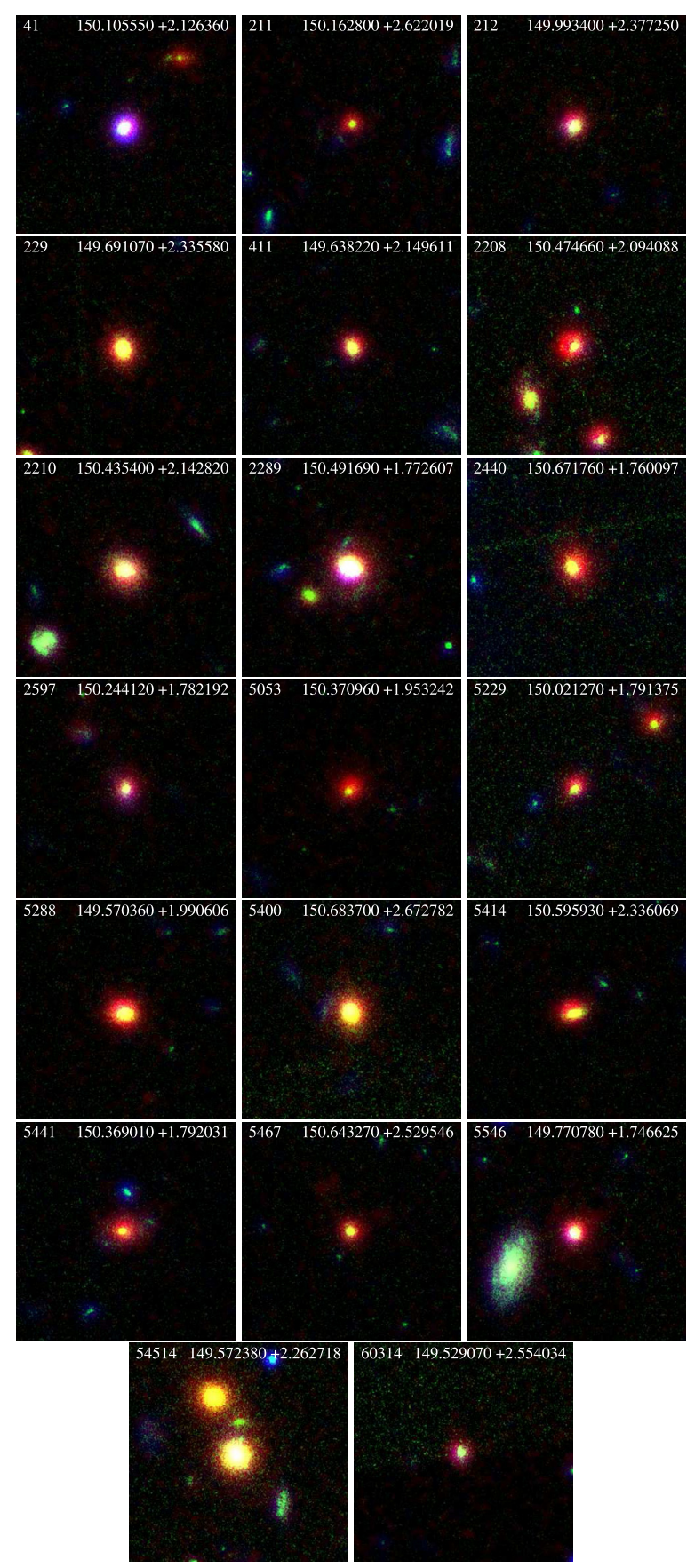

Fig. 12. Type-2 QSOs hosts classified as bulge-dominated galaxies. The images are $10^{\prime \prime}$ on a side. They were obtained by combining Subaru $B$-band (blue), ACS F814W (green), and CFHT Ks (red) images. XID and coordinates of the source are reported at the top of each image.

by Scarlata et al. (2007), in the third panel a significant fraction of our hosts are outside the contours mainly due to larger asymmetry values than observed for galaxies of the same morphological class. This could be interpreted as an indication of a higher fraction of disturbed morphologies in our sample of Type-2 QSO host galaxies than normally observed in a magnitude limited sample of normal galaxies. Furthermore, our hosts tend to be highly concentrated and $\approx 65 \%$ of them have $\mathrm{C}>3$, a range in which elliptical and bulge-dominated galaxies are located. This is not surprising since they are mostly massive galaxies that usually have a consistent bulge already in place. Silverman et al. (2008) also found that for X-ray selected

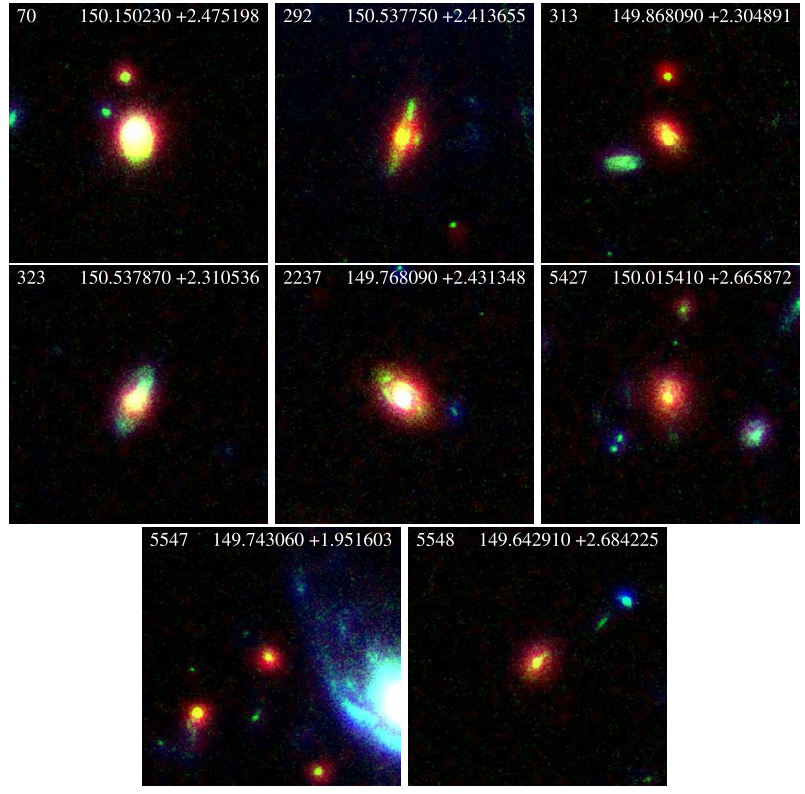

Fig. 13. Type-2 QSOs hosts classified as disk-dominated galaxies. The images are $10^{\prime \prime}$ on a side. They were obtained by combining Subaru $B$-band (blue), ACS F814W (green), and CFHT Ks (red) images. XID and coordinates of the source are reported at the top of each image.

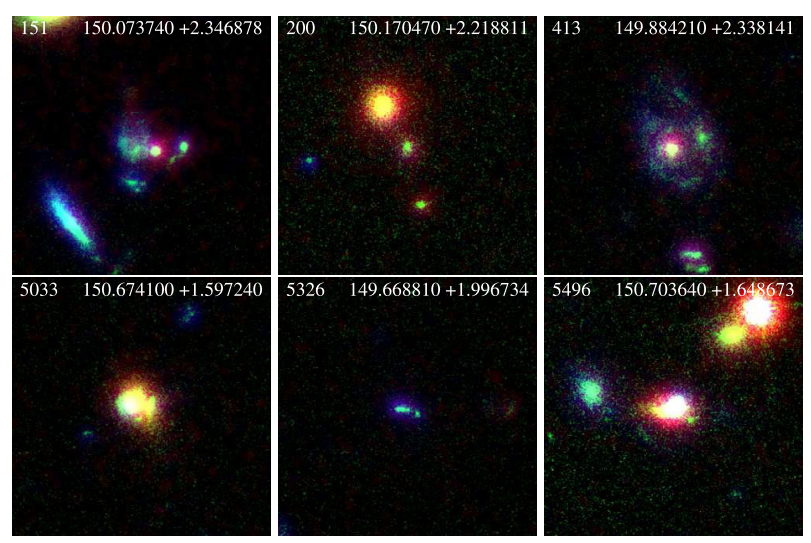

Fig. 14. Type-2 QSOs hosts classified as possible-merger. The images are $10^{\prime \prime}$ on a side. They were obtained by combining Subaru $B$-band (blue), ACS F814W (green), and CFHT Ks (red) images. XID and coordinates of the source are reported at the top of each image.

AGN of lower luminosity $\left(\log L_{0.5-10 \mathrm{keV}}<43.7 \mathrm{erg} \mathrm{s}^{-1}\right)$ at $z<1$ the majority of the host galaxies are bulge dominated. We note that in the morphological analysis presented in this paper, we did not attempt to subtract any possible residual point-like source at the center of the galaxy. If present, these point sources would bias the concentration measurements towards higher values: Gabor et al. (2009) found that this is the case for a sample of $\approx 200 \mathrm{X}$-ray selected AGN at $0.3<z<1$ in the COSMOS field (see their Fig. 12). The AGN sample of Gabor et al. (2009) was, on average, less obscured than the $N_{\mathrm{H}}>10^{22} \mathrm{~cm}^{-2}$ objects we present, hence the likelihood that a residual point-like source is present in the ACS images is higher. We plan to study this possible bias in detail in future work.

As reported above, we were able to detect signs of recent mergers in only $\approx 20 \%$ of the hosts of Type- 2 QSOs at $z<1.2$. The lack of morphological merger signatures for the majority of the objects should be treated with caution. The ACS images could simply be not deep enough to detect faint surface 

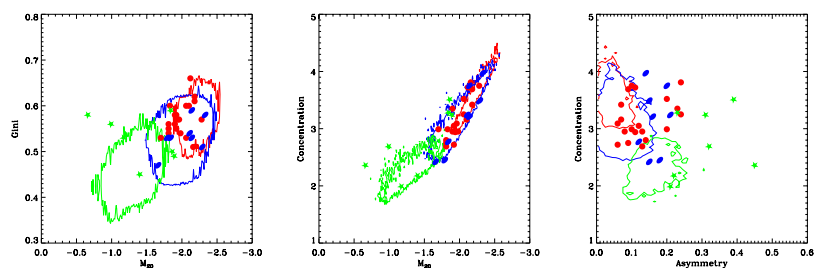

Fig. 15. Location of the hosts morphologically classified as bulgedominated (filled circles), disk-dominated (filled ellipse), possiblemergers (filled stars) in three planes defined using the four non-parametric measurements of the light distribution: asymmetry, concentration, M 20, Gini coefficient. The contours include $99 \%$ of the Scarlata et al. (2007) galaxies with morphological classification as bulge-dominated (red), disk-dominated (blue), and possible-mergers (green).

brightness features, as tidal tails or shells, to trace past interactions. For example Ramos Almeida et al. (2011), using deep optical images, found that $\approx 80 \%$ of a complete sample of $z<0.7$ narrow line radio galaxies show signs of interactions based on the detection of faint morphological features. In addition, some models (e.g., Di Matteo et al. 2005) predict that the quasar phase occurs towards the end of the merging process, and at that point the final galaxy should be mostly relaxed and have low values of asymmetry (e.g., Conselice 2003). In this scenario, it becomes difficult to test merging-induced quasar fueling models by studying the morphological appearance of the host galaxies. At the same time, this hypothesis also opens another possible approach: a major merger would most likely form spheroidal galaxies and large disks should usually be destroyed ${ }^{9}$. As described above, we find that the majority of the Type-2 QSO hosts at $z<1.2$ are bulge-dominated galaxies and that a few of them contain disks. In a study of the morphology of the hosts of 140 AGN in the XMM-COSMOS survey, Cisternas et al. (2011) found that: a) there is no significant difference in the distortion fractions between active and inactive galaxies; b) $65 \%$ of the AGN hosts are disk dominated. On the basis of these findings, they concluded that major mergers are not the trigger of $\mathrm{BH}$ accretion. We note that the vast majority of the Cisternas et al. (2011) AGN sample has lower X-ray luminosities than the Type-2 QSOs presented in this paper: only 18 sources (13\%) of their sample have $L_{\mathrm{X}}>10^{44} \mathrm{erg} \mathrm{s}^{-1}$ and these are mostly unobscured QSOs. The different fraction of disk galaxies between the two studies could be connected to different triggering mechanisms of accretion activity at low and high AGN luminosities (e.g., Hopkins \& Hernquist 2009).

In Fig. 16, we compared the distribution of Eddington ratios for the three morphological classes. The range of values of this statistic is limited, but bulge-dominated galaxies tend to host AGN with low Eddington ratios $(\lambda<0.1)$, while disk-dominated or merging galaxies at their centers contain BHs accreting at high Eddington ratios $(\lambda>0.1)$. The mean $\mathrm{BH}$ mass of the bulge-dominated sample is $\left\langle M_{\mathrm{BH}}\right\rangle \sim 4 \times 10^{8} M_{\odot}$, while for the mergers $\left\langle M_{\mathrm{BH}}\right\rangle \sim 1 \times 10^{8} M_{\odot}$. The distributions in Fig. 16 are therefore consistent with the general finding that the lowest mass BHs are the fastest accretors (McLure \& Dunlop 2002; Netzer \& Trakhtenbrot 2007).

\footnotetext{
9 Some simulations (e.g., Barnes \& Hernquist 1996; Springel \& Hernquist 2005) show that mergers of gas-rich galaxies could produce a disk-dominated remnant but only for particular orientations of the angular momentum vectors. These conditions are usually not related to substantial BH growth (Hopkins \& Hernquist 2009).
}

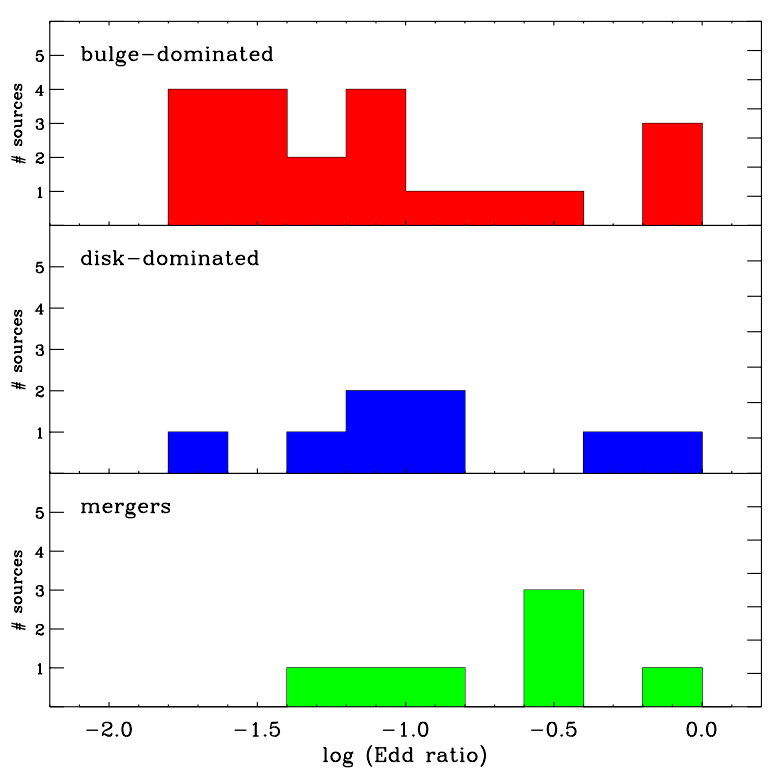

Fig. 16. Distribution of Eddington ratios for the hosts of Type2 QSOs morphologically classified as bulge-dominated (top panel), disk-dominated (middle-panel), and possible mergers (bottom panel).

\section{Conclusions}

We have explored the relationships between SMBH accretion in obscured quasars and the physical properties of their host galaxies. The sample of 142 Type-2 QSOs was selected from those considered for the X-ray spectral analysis in the XMM-COSMOS survey. The bulk of this quasar sample is located in the redshift range $1<z<2.5$, which includes the peaks of both the global SFR and AGN activity. The obscured quasars presented in the paper have a mean bolometric luminosity of $L_{\mathrm{bol}}=8 \times 10^{45} \mathrm{erg} \mathrm{s}^{-1}$, a regime where several models invoke major mergers of gas-rich galaxies as the main fueling mechanism of the central SMBH.

A key ingredient of this work has been the ability to use an AGN+host galaxy SED decomposition technique to put reliable constraints on the stellar masses and SFRs of the hosts. This has been possible for the excellent multi-wavelength coverage in the COSMOS field.

The main results of this work are the following:

- Type-2 QSOs reside almost exclusively in massive galaxies: more than $80 \%$ of their hosts have $M_{\star}>10^{10} M_{\odot}$. The fraction of galaxies hosting Type-2 QSOs monotonically increases with stellar masses going from $0.02 \%$ at $M_{\star} \approx 8 \times$ $10^{9} M_{\odot}$ to $\approx 0.6 \%$ at $M_{\star} \approx 10^{11} M_{\odot}$.

- The rest-frame colors of their host galaxies are mostly between those of the blue and red galaxy population. We confirm that this is the effect of a luminosity-selected rather than a mass-selected sample. Furthermore, the classification of the host galaxies based on their rest-frame colors is subject to the effect of dust reddening: $\approx 20 \%$ of the host galaxies with red or intermediate rest-frame colors are dusty star-forming objects.

- At $z \sim 1, \approx 62 \pm 7 \%$ of Type-2 QSOs are actively forming stars and this fraction increases with redshift, $\approx 71 \pm 3 \%$ at $z \sim 2$, and $100 \%$ at $z \sim 3$.

- We find that the redshift evolution of the specific SFR for the hosts of Type-2 QSOs is in excellent agreement with that observed for star-forming galaxies in the redshift range 
$1<z<3$ : the average SSFR increase by a factor $\approx 3.6$ going from $z \sim 1$ to $z \sim 2$, and remains constant at higher redshifts.

- A morphological analysis of the ACS images has indicated that the majority of the host galaxies are bulge dominated, there being a few cases of disk galaxies or mergers. This lack of merger signatures cannot be automatically translated as proof that mergers are not playing an important role in the accretion history of these quasars. Several models indeed assume that the quasar phase occurs toward the end of the merging process, and at that point we expect the host galaxy to be mostly relaxed with low values of asymmetry. Numerical simulations also predict that large disks are destroyed during a major merger, therefore detecting large number of disks could place some strong constraint on the occurrence of a major merger. With the current data, we have detected clear disks in only a few cases.

- We find that bulge-dominated galaxies tend to host Type2 QSOs with low Eddington ratios $(\lambda<0.1)$, while diskdominated or merging galaxies have at their centers BHs accreting at high Eddington ratios $(\lambda>0.1)$.

The morphological analysis presented in this paper is based on F814W ACS images, which corresponds to the rest-frame UV at the average redshift of our galaxies. This implies that we are mainly mapping regions of recent star-formation. It will be extremely interesting to repeat this analysis using future nearIR observations from space that will provide high resolution images in the optical rest-frame. The CANDELS HST Multicycle Treasury Program (Grogin et al. 2011; Koekemoer et al. 2011) will image with WFC3/HST part of the COSMOS field and therefore provide a less biased view of the morphology of these Type-2 QSOs hosts. It would be also interesting to estimate the nebular continuum contribution to the UV SED of Type-2 quasars host galaxies. This would be possible using NIR spectroscopy to derive $\mathrm{H} \beta$ flux and the line of sight reddening for each quasar (see e.g., Tadhunter et al. 2002). Finally, the ongoing Herschel survey of the COSMOS field, as well as other patches of the sky, will improve the mid and far-infrared coverage, a crucial wavelength range to measure the SFR of AGN hosts.

Acknowledgements. We are grateful to Hagai Netzer for inspiring discussions and to Giulia Rodighiero for providing the Herschel curves in Fig. 9 in electronic format. We thank the referee, Montse Villar-Martin, for a careful reading of the manuscript and useful comments. This work is based on observations made with ESO Telescopes at the La Silla/Paranal Observatories under the zCOSMOS Large Programme 175.A-0839; the XMM-Newton satellite, an ESA science mission with instruments and contributions directly funded by ESA Member States and the US (NASA); the Magellan Telescope, operated by the Carnegie Observatorie and the MMT Observatory, a joint facility of the University of Arizona and the Smithsonian Institution; the Subaru Telescope, operated by the National Astronomical Observatory of Japan; and the NASA/ESA Hubble Space Telescope, operated at the Space Telescope Science Institute, which is operated by AURA Inc, under NASA contract NAS 5-26555. Support from the Italian Space Agency (ASI) under the contracts ASI-INAF I/088/06/0 and I/009/10/0 is acknowledged. G.H. and M.S. acknowledge support by the German Deutsche Forschungsgemeinschaft, DFG Leibniz Prize (FKZ HA 1850/28-1).

\section{References}

Abraham, R. G., van den Bergh, S., \& Nair, P. 2003, ApJ, 588, 218 Archibald, E. N., Dunlop, J. S., Hughes, D. H., et al. 2001, MNRAS, 323, 417 Avni, Y. 1976, ApJ, 210, 642

Barnes, J. E., \& Hernquist, L. 1996, ApJ, 471, 115

Barger, A. J., Cowie, L. L., Mushotzky, R. F., et al. 2005, AJ, 129, 578

Bell, E. F., Wolf, C., Meisenheimer, K., et al. 2004, ApJ, 608, 752

Best, P. N., Kauffmann, G., Heckman, T. M., et al. 2005, MNRAS, 362, 25

Blanton, M. R., Hogg, D. W., Bahcall, N. A., et al. 2003, ApJ, 594, 186

Balogh, M. L., Morris, S. L., Yee, H. K. C., et al. 1999, ApJ, 527, 54
Bianchi, S., Guinazzi, M., Matt, G., \& Fonseca Bonilla, N. 2007, A\&A, 467, L19

Brammer, G. B., Whitaker, K. E., van Dokkum, P. G., et al. 2009, ApJ, 706, L173

Brinchmann, J., Charlot, S., White, S. D. M., et al. 2004, MNRAS, 351, 1151

Brusa, M., Comastri, A., Gilli, R., et al. 2009a, ApJ, 693, 8

Brusa, M., Fiore, F., Santini, P., et al. 2009b, A\&A, 507, 1277

Brusa, M., Civano, F., Comastri, A., et al. 2010, ApJ, 716, 348

Bruzual, G., \& Charlot, S. 2003, MNRAS, 344, 1000

Calzetti, D., Armus, L., Bohlin, R. C., et al. 2000, ApJ, 533, 682

Cappelluti, N., Brusa, M., Hasinger, G., et al. 2009, A\&A, 497, 635

Cardamone, C. N., Urry, C. M., Schawinski, K., et al. 2010, ApJ, 721, L38

Cassata, P., Cimatti, A., Kurk, J., et al. 2008, A\&A, 483, L39

Cen, R. 2011, ApJ, submitted [arXiv: 1102 . 0262]

Chabrier, G. 2003, ApJ, 586, L133

Chaudhary, P., Brusa, M., Hasinger, G., Merlini, A., \& Comastri, A. 2010, A\&A, 518, A58

Ciotti, L., Ostriker, J. P., \& Proga, D. 2010, ApJ, 717, 708

Cirasuolo, M., McLure, R. J., Dunlop, J. S., et al. 2007, MNRAS, 380, 585

Cisternas, M., Jahnke, K., Inskip, K. J., et al. 2011, ApJ, 726, 57

Conselice, C. J. 2003, ApJS, 147, 1

Croton, D. J., Sprigel, V., White, S. D. M., et al. 2006, MNRAS, 365, 11

Daddi, E., Cimatti, A., Renzini, A., et al. 2004, ApJ, 617, 746

Daddi, E., Dickinson, M., Morrison, G., et al. 2007, ApJ, 670, 156

Dawson, S., Stern, D., Bunker, A. J., Spinrad, H., \& Dey, A. 2001, AJ, 122, 598

Della Ceca, R., Braito, V., Beckmann, V., et al. 2003, A\&A, 406, 555

Dickey, J. M., \& Lockman, F. J. 1990, ARA\&A, 28, 215

Dickinson, M., Papovich, C., Ferguson, H. C., \& Budavari, T. 2003, ApJ, 587, 25

Dickson, R., Tadhunter, C., Shaw, M., Clark, N., \& Morganti, R. 1995, MNRAS, 273, L29

Di Matteo, T., Springel, V., \& Hernquist, L. 2005, Nature, 433, 604

Elbaz, D., Daddi, E., Le Borgne, D., et al. 2007, A\&A, 468, 33

Ferrarese, L., \& Merritt, D. 2000, ApJ, 539, L9

Fiore, F., Brusa, M., Cocchia, F., et al. 2003, A\&A, 409, 79

Fontana, A., Pozzetti, L., Donnarumma, I., et al. 2004, A\&A, 459, 745

Franzetti, P., Scodeggio, M., Garilli, B., et al. 2007, A\&A, 465, 711

Gabor, J. M., Impey, C. D., Jahnke, K., et al. 2009, ApJ, 691, 705

Gandhi, P., Horst, H., Smette, A., et al. 2009, A\&A, 502, 457

Gebhardt, K., Kormendy, J., Ho, L. C., et al. 2000, ApJ, 539, L13

Genzel, R., Burkert, A., Bouche, N., et al. 2008, ApJ, 687, 59

Georgakakis, A., Nandra, K., Yan, R., et al. 2008, MNRAS, 385, 2049

Giallongo, E., Salimbeni, S., Menci, N., et al. 2005, ApJ, 622, 116

Gilli, R., Comastri, A., \& Hasinger, G. 2007, A\&A, 463, 79

Gilli, R., Vignali, C., Mignoli, M., et al. 2010, A\&A, 519, A92

Gonzalez, V., Labbe, I., Bouwens, R. J., et al. 2010, ApJ, 713, 115

Graham, A. W., Erwin, P., Caon, N., \& Trujillo, I. 2001, ApJ, 563, L11

Granato, G. L., De Zotti, G., Silva, L., Bressan, A., \& Danese, L. 2004, ApJ, 600,580

Granato, G. L., Silva, L., Lapi, A., et al. 2006, MNRAS, 368, L72

Grogin, N. A., Kocevski, D. D., Faber, S. M., et al. 2011, ApJS, submitted [arXiv: 1105.3753]

Häring, N., \& Rix, H.-W. 2004, ApJ, 604, L89

Hasinger, G. 2004, Nucl. Phys. B, 132, 86

Hasinger, G. 2008, A\&A, 490, 905

Hasinger, G., Miyaji, T., \& Schmidt, M. 2005, A\&A, 441, 417

Hickox, R. C., Jones, C., Forman, W. R., et al. 2009, ApJ, 696, 891

Hogg, D. W., Blanton, M., Strateva, I., et al. 2002, AJ, 124, 646

Hopkins, P. F., \& Hernquist, L. 2009, ApJ, 694, 599

Hopkins, P. F., Hernquist, L., Cox, T. J., Robertson, B., \& Springel, V. 2006, ApJS, 163, 50

Hopkins, P. F., Richards, G. T., \& Hernquist, L. 2007, ApJ, 654, 731

Hopkins, P. F., Hickox, R., Quataert, E., \& Hernquist, L. 2009, MNRAS, 398, 333

Ilbert, O., Salvato, M., Le Floc'h, E., et al. 2010, ApJ, 709, 644

Iwasawa, K., \& Taniguchi, Y. 1993, ApJ, 413, 15

Jahnke, K., \& Macció, A. V. 2011, ApJ, 734, 92

Jogee, S. 2004, Physics of Active Galactic Nuclei at all Scales, 693, 143

Johansson, P. H., Naab, T., \& Burkert, A. 2009, ApJ, 690, 802

Karim, A., Schinnerer, E., Martinez-Sansigre, A., et al. 2011, ApJ, 730, 61

Kaspi, S., Smith, P. S., Netzer, H., et al. 2000, ApJ, 533, 631

Kauffmann, G., \& Haehnelt, M. G. 2000, MNRAS, 311, 576

Kauffmann, G., Heckman, T. M., Tremonti, C., et al. 2003, MNRAS, 346, 1055

Koekemoer, A., Aussel, H., Calzetti, D., et al. 2007, ApJS, 172, 196

Koekemoer, A., Faber, S. M., Ferguson, H. C., et al. 2011, ApJS, submitted [arXiv: 1105.3754]

Kollmeier, J. A., Onken, C. A., Kochanek, C. S., et al. 2006, ApJ, 648, 128

Kormendy, J., \& Richstone, D. 1995, ARA\&A, 33, 581

Krumpe, M., Lamer, G., Corral, A., et al. 2008, A\&A, 483, 415 
Lanzuisi, G., Piconcelli, E., Fiore, F., et al. 2009, A\&A, 498, 67 Lapi, A., Shankar, F., Mao, J., et al. 2006, ApJ, 650, 42 Lehmer, B. D., Brandt, W. N., Alexander, D. M., et al. 2007, ApJ, 657, 681 Lilly, S. J., Le Brun, V., Maier, C., et al. 2009, ApJS, 184, 218 Lotz, J. M., Primack, J., \& Madau, P. 2004, AJ, 128, 163 Lusso, E., Comastri, A., Vignali, C., et al. 2010, A\&A, 512, A34 Luzt, D., Mainieri, V., Rafferty, D., et al. 2010, ApJ, 712, 1287 Magorrian, J., Tremaine, S., Richstone, D., et al. 1998, AJ, 115, 2285 Mainieri, V., Bergeron, J., Hasinger, G., et al. 2002, A\&A, 393, 425 Mainieri, V., Hasinger, G., Cappelluti, N., et al. 2007, ApJS, 179, 95 Maiolino, R., Marconi, A., Salvati, M., et al. 2001, A\&A, 365, 28

Maiolino, R., Neri, R., Beelen, A., et al. 2007, A\&A, 468, 979 Marconi, A., \& Hunt, L. K. 2003, ApJ, 589, L21

Marconi, A., Risaliti, G., Gilli, R., et al. 2004, MNRAS, 351, 169

Martin, D. C., Wyder, T. K., Schiminovich, D., et al. 2007, ApJS, 173, 342

Martínez-Sansigre, A., Rawlings, S., Lacy, M., et al. 2006, MNRAS, 370, 1479

Mateos, S., Barcons, X., Carrera, F. J., et al. 2005, A\&A, 433, 855

McCarthy, P. J. 1993, ARA\&A, 31, 639

McLure, M. J., \& Dunlop, J. S. 2002, MNRAS, 331, 795

Menci, N., Fontana, A., Giallongo, E., \& Salimbeni, S. 2005, ApJ, 632, 49

Merloni, A., Rudnick, G., \& Di Matteo, T. 2004, MNRAS, 354, L37

Merloni, A., Bongionro, A., Bolzonella, M., et al. 2010, ApJ, 708, 137

Merritt, D., \& Ferrarese, L. 2001, MNRAS, 320, L30

Monaco, P., \& Fontanot, F. 2005, MNRAS, 359, 283

Morrison, R., \& McCammon, D. 2001, AJ, 556, L75

Moustakas, J., Kennicutt, R. C., \& Tremonti, C. A. 2006, ApJ, 642, 775

Nandra, K., Georgakakis, A., Willmer, C. N., et al. 2007, ApJ, 660, L11

Netzer, H., \& Trakhtenbrot, B. 2007, ApJ, 654, 754

Netzer, H., Mainieri, V., Rosati, P., \& Trakhtenbrot, B. 2006, A\&A, 453, 525

Netzer, H., Lutz, D., Schweitzer, M., et al. 2007, ApJ, 666, 806

Noeske, K. G., Weiner, B. J., Faber, S. M., et al. 2007a, ApJ, 660, L43

Noeske, K. G., Faber, S. M., Weiner, B. J., et al. 2007b, ApJ, 660, L47

Norman, C., Hasinger, G., Giacconi, R., et al. 2002, ApJ, 571, 218

Pannella, M., Carilli, C. L., Daddi, E., et al. 2009, ApJ, 698, L116

Peng, C. Y. 2007, ApJ, 671, 1098

Peng, Y., Lilly, S. J., Kovac, K., et al. 2010, ApJ, 721, 193

Perola, G. C., Puccetti, S., Fiore, F., et al. 2004, A\&A, 421, 491

Polletta, M., Weedman, D., Hoenig, S., et al. 2008, ApJ, 675, 960

Pozzetti, L., Bolzonella, M., Lamareille, F., et al. 2007, A\&A, 474, 443

Pozzetti, L., Bolzonella, M., Zucca, E., et al. 2010, A\&A, 523, A13

Prevot, M. L., Lequeux, J., Prevot, L., Maurice, E., \& Rocca-Volmerange, B. 1984, A\&A, 132, 389

Ramos Almeida, C., Tadhunter, C. N., Inskip, K. J., et al. 2011, MNRAS, 410, 1550

Reuland, M., Roettgering, H., van Breugel, W., \& De Breuck, C. 2004, MNRAS, 353, 377

Reyes, R., Zakamska, N. L., Strauss, M. A., et al. 2008, AJ, 136, 2373

Richards, G. T., Lacy, M., Storrie-Lombardi, L. J., et al. 2006, ApJS, 166, 470

Rodighiero, G., Cimatti, A., Gruppioni, C., et al. 2010, A\&A, 518, L25

Rovilos, E., \& Georgantoupoulos, I. 2007, A\&A, 475, 115

Salim, S., Charlot, S., Rich, R. M., et al. 2005, ApJ, 619, L39

Salpeter, E. E. 1955, ApJ, 121, 161

Salvato, M., Hasinger, G., Ilbert, O., et al. 2009, ApJ, 690, 1250

Scarlata, C., Carollo, C. M., Lilly, S., et al. 2007, ApJS, 172, 406

Scoville, N., Aussel, H., Brusa, M., et al. 2007, ApJS, 172,

Shao, L., Lutz, D., Nordon, R., et al. 2010, A\&A, 518, L26

Schawinski, K., Virani, S., Simmons, B., et al. 2009, ApJ, 692, L19

Schawinski, K., Urry, C. M., Virani, S., et al. 2010, ApJ, 711, 284

Silverman, J. D., Mainieri, V., Lehmer, B. D., et al. 2008, ApJ, 675, 1025

Silverman, J. D., Lamareille, F., Maier, C., et al. 2009, ApJ, 696, 396

Simpson, C. 2005, MNRAS, 360, 565

Somerville, R. S., Primack, J. R., \& Faber, S. M. 2001, MNRAS, 320, 504

Springel, V., \& Hernquist, L. 2005, ApJ, 622, L9

Springel, V., Di Matteo, T., \& Hernquist, L. 2005, MNRAS, 361, 776

Stark, D. P., Ellis, R. S., Bunker, A., et al. 2009, ApJ, 697, 1493

Steffen, A. T., Barger, A. J., Cowie, L. L., Mushotzky, R. F., \& Yang, Y. 2003, ApJ, 596, L23

Stern, D., Moran, E. C., Coil, A. L., et al. 2002, ApJ, 568, 71

Strateva, I., Ivezic, Z., Knapp, G. R., et al. 2001, AJ, 122, 186

Szokoly, G. P., Bergeron, J., Hasinger, G., et al. 2004, ApJS, 155, 271

Tadhunter, C., Dickson, R., Morganti, R., et al. 2002, MNRAS, 330, 977

Tozzi, P., Gilli, R., Mainieri, V., et al. 2006, A\&A, 451, 457

Treister, E., Krolik, J. H., \& Dullemond, C. 2008, ApJ, 679, 140

Tremaine, S., Gebhardt, K., Bender, R., et al. 2002, ApJ, 574, 740
Trump, J. R., Impey, C. D., Elvis, M., et al. 2009, ApJ, 696, 1195

Ueda, Y., Akiyama, M., Ohta, K., \& Miyaji, T. 2003, ApJ, 598, 886

Vasudevan, R. V., \& Fabian, A. C. 2007, MNRAS, 381, 1235

Vernet, J., Fosbury, R. A. E., Villar-Martin, M., et al. 2001, A\&A, 366, 7

Vignali, C., Alexander, D. M., Gilli, R., \& Pozzi, F. 2010, MNRAS, 404, 48

Xue, Y. Q., Brandt, W. N., Luo, B., et al. 2010, ApJ, 720, 368

Weiner, B. J., Philips, A. C., Faber, S. M., et al. 2005, ApJ, 620, 595

Wilkins, S. M., Trentham, N., \& Hopkins, A. M. 2008, MNRAS, 385, 687

Williams, R. J., Quadri, R. F., Franx, M., van Dokkum, P., \& Labbe, I. 2009 ApJ, 691, 1879

Willmer, C. N. A., Faber, S. M., Koo, D. C., et al. 2006, ApJ, 647, 853

Winter, L. M., Lewis, K. T., Koss, M., et al. 2010, ApJ, 710, 503

Worthey, G., \& Ottaviani, D. L. 1997, ApJS, 111, 377

Zakamska, N. L., Strauss, M. A., Krolik, J. H., et al. 2003, AJ, 126, 2125

Zakamska, N. L., Schmidt, G. D., Smith, P. S., et al. 2005, AJ, 129, 1212

1 ESO, Karl-Schwarschild-Strasse 2, 85748 Garching bei München, Germany

e-mail: vmainier@eso.org

2 Max-Planck-Institute für Extraterrestrische Physik, Postfach 1312, 85741 Garching bei München, Germany

3 Excellence Cluster Universe, TUM, Boltzmannstr. 2, 85748 Garching bei München, Germany

4 Institute for Astronomy, ETH Zurich, 8093 Zurich, Switzerland

5 ICREA and Institut de Ciències del Cosmos (ICC), Universitat de Barcelona (IEEC-UB), Martí i Franquès, 1, 08028 Barcelona, Spain

6 Space Telescope Science Institute, Baltimore, Maryland 21218, USA

7 INAF - Osservatorio Astronomico di Bologna, via Ranzani 1, 40127 Bologna, Italy

8 Institute for the Physics and Mathematics of the Universe (IPMU), University of Tokyo, Kashiwanoha 5-1-5, Kashiwa-shi, Chiba 2778568, Japan

9 INAF - Osservatorio Astrofisico di Arcetri, Largo Enrico Fermi 5, 50125 Firenze, Italy

10 Laboratoire d'Astrophysique de Marseille, Marseille, France

11 Dipartimento di Astronomia, Universita‘ di Bologna, via Ranzani 1, 40127 Bologna, Italy

12 Max-Planck-Institute für Plasma Physis, Boltzmann Strasse 2, 85748 Garching bei München, Germany

13 Laboratoire d'Astrophysique de Toulouse-Tarbes, Université de Toulouse, CNRS, 14 avenue Édouard Belin, 31400 Toulouse, France

14 Dipartimento di Astronomia, Universita di Padova, Padova, Italy

15 INAF-IASF Milano, Milan, Italy

16 SUPA, Institute for Astronomy, The University of Edinburgh, Royal Observatory, Edinburgh EH9 3HJ, UK

17 INAF - Osservatorio Astronomico di Brera, Milan, Italy

18 Max-Planck-Institute für Astrophysik, 85748 Garching bei München, Germany

19 Dipartimento di Astronomia, Universita di Padova, Padova, Italy

20 California Institute of Technology, MC 105-24, 1200 East California Boulevard, Pasadena, CA 91125, USA

21 Harvard-Smithsonian Center for Astrophysics, 60 Garden St., Cambridge, MA 02138, USA

22 Steward Observatory, University of Arizona, 933 North Cherry Avenue, Tucson, AZ 85721, USA

23 INAF - Osservatorio Astronomico di Roma, via Frascati 33, 00040 Monteporzio-Catone, Italy

24 Research Center for Space and Cosmic Evolution, Ehime University, Bunkyo-cho 2-5, Matsuyama 790-8577, Japan

25 UCO/Lick Observatory, University of California, Santa Cruz, CA 95064, USA

26 AIM, Unité Mixte de Recherche CEA CNRS, Université Paris VII, UMR n158, Paris France

27 CEA-Saclay, Service d'Astrophysique, Orme des Merisiers, Bât.709, 91191 Gif-sur-Yvette, France 
A\&A 535, A80 (2011)

Table 1. SED of Type-2 QSOs.

\begin{tabular}{|c|c|c|c|c|c|c|c|c|}
\hline XID & $\begin{array}{c}\log L_{\mathrm{X}}[0.5-10 \mathrm{keV}] \\
\left(\mathrm{erg} \mathrm{s}^{-1}\right)\end{array}$ & $\begin{array}{l}\log N_{\mathrm{H}} \\
\left(\mathrm{cm}^{-2}\right) \\
\end{array}$ & $z$ & Survey & $\begin{array}{c}\log M \star \\
\left(M_{\odot}\right) \\
\end{array}$ & $\begin{array}{c}S F R \\
\left(M_{\odot} \mathrm{yr}^{-1}\right) \\
\end{array}$ & $\begin{array}{l}M_{U} \\
(\mathrm{AB}) \\
\end{array}$ & $\begin{array}{c}M_{B} \\
(\mathrm{AB}) \\
\end{array}$ \\
\hline 41 & 45.20 & $22.1_{22.1}^{22.5}$ & 0.962 & s & $10.2_{10.2}^{10.2}$ & $79.1_{78.3}^{79.4}$ & -21.4 & -22.0 \\
\hline 50 & 45.44 & $22.5_{22.3}^{22.6}$ & 2.941 & s & $9.8_{9.8}^{9.8}$ & $157.8_{157.8}^{158.9}$ & -21.8 & -22.2 \\
\hline 70 & 44.51 & $23.2_{23.1}^{23.4}$ & 0.688 & s & $10.8_{10.8}^{10.8}$ & $94.8_{94.8}^{94.8}$ & -20.6 & -21.7 \\
\hline 97 & 44.53 & $22.4_{22.2}^{22.5}$ & 1.300 & $\mathrm{p}$ & $10.7_{10.7}^{10.9}$ & $64.3_{64.3}^{64.0}$ & -20.9 & -21.8 \\
\hline 105 & 44.68 & $22.7_{22.3}^{22.9}$ & 1.600 & $\mathrm{p}$ & $10.7_{10.7}^{10.7}$ & $4.3_{4.3}^{4.3}$ & -19.8 & -20.9 \\
\hline 106 & 44.16 & $22.6_{22.5}^{22.8}$ & 1.260 & $\mathrm{p}$ & $10.8_{10.8}^{10.8}$ & $0.5_{0.5}^{0.5}$ & -19.1 & -20.5 \\
\hline 107 & 44.77 & $22.0_{21.3}^{22.5}$ & 1.530 & $\mathrm{p}$ & $10.4_{10.3}^{10.0}$ & $33.0_{32.2}^{33.0}$ & -21.9 & -22.5 \\
\hline 108 & 44.44 & $22.3_{21.4}^{22.6}$ & 1.570 & $\mathrm{p}$ & $10.5_{10.5}^{10.5}$ & $84.7_{83.8}^{86.5}$ & -20.4 & -21.4 \\
\hline 122 & 45.12 & $23.0_{22.8}^{23.4}$ & 2.418 & s & $10.6_{10.6}^{10.6}$ & $0.7_{0.7}^{0.8}$ & -20.1 & -21.2 \\
\hline 138 & 44.85 & $22.7_{22.6}^{22.9}$ & 2.610 & $\mathrm{p}$ & $11.0_{10.6}^{11.0}$ & $305.5_{296.5}^{946.2}$ & -22.4 & -23.2 \\
\hline 144 & 44.96 & $22.6_{22.3}^{22.9}$ & 2.930 & $\mathrm{p}$ & $11.5_{11.5}^{11.5}$ & $32.7_{32.5}^{32.7}$ & -22.2 & -23.3 \\
\hline 151 & 44.10 & $22.7_{22.4}^{22.9}$ & 0.797 & s & $10.6_{10.6}^{10.6}$ & $113.2_{110.9}^{114.0}$ & -21.9 & -22.7 \\
\hline 159 & 44.43 & $22.1_{21.2}^{22.5}$ & 2.230 & $\mathrm{p}$ & $11.5_{11.5}^{11.5}$ & $116.9_{116.1}^{118.3}$ & -21.0 & -22.2 \\
\hline 181 & 44.58 & $22.2_{22.0}^{22.4}$ & 1.720 & $\mathrm{p}$ & $11.1_{11.1}^{11.2}$ & $63.1_{62.4}^{63.5}$ & -20.4 & -21.6 \\
\hline 182 & 44.91 & $22.9_{22.7}^{23.0}$ & 2.040 & $\mathrm{p}$ & $10.9_{10.9}^{11.1}$ & $0.6_{0.6}^{1.0}$ & -21.1 & -22.3 \\
\hline 194 & 44.40 & $22.7_{22.4}^{23.0}$ & 1.456 & s & $10.6_{10.4}^{10.6}$ & $65.3_{64.7}^{67.5}$ & -20.8 & -21.7 \\
\hline 200 & 44.07 & $22.7_{22.3}^{23.0}$ & 1.156 & s & $10.3_{10.3}^{10.3}$ & $22.2_{22.2}^{22.5}$ & -19.7 & -20.7 \\
\hline 209 & 44.55 & $22.9_{22.5}^{23.3}$ & 2.000 & $\mathrm{p}$ & $11.0_{10.8}^{11.0}$ & $33.0_{14.2}^{33.7}$ & -20.3 & -21.4 \\
\hline 211 & 44.02 & $22.2_{22.0}^{22.4}$ & 1.188 & s & $10.5_{10.5}^{10.5}$ & $55.3_{54.7}^{55.3}$ & -20.0 & -21.0 \\
\hline 212 & 44.55 & $23.4_{23.2}^{24.3}$ & 0.931 & s & $10.7_{10.4}^{10.7}$ & $13.2_{2.7}^{13.3}$ & -20.1 & -21.2 \\
\hline 229 & 44.03 & $22.2_{21.8}^{22.5}$ & 0.864 & s & $11.1_{11.1}^{11.4}$ & $1.6_{1.6}^{1.6}$ & -20.2 & -21.5 \\
\hline 233 & 44.59 & $22.7_{22.5}^{22.9}$ & 1.930 & $\mathrm{p}$ & $10.6_{10.5}^{10.1}$ & $76.6_{33.5}^{84.9}$ & -20.4 & -21.4 \\
\hline 235 & 44.79 & $22.6_{22.4}^{22.8}$ & 2.220 & $\mathrm{p}$ & $11.5_{11.5}^{11.5}$ & $370.7_{370.7}^{376.7}$ & -22.2 & -23.3 \\
\hline 247 & 44.49 & $23.0_{22.6}^{23.3}$ & 1.950 & $\mathrm{p}$ & $10.8_{10.5}^{10.8}$ & $19.2_{7.9}^{19.5}$ & -20.3 & -21.3 \\
\hline $276^{10}$ & 45.28 & $22.8_{22.7}^{23.0}$ & 2.620 & $\mathrm{p}$ & $10.8_{10.8}^{10.8}$ & $790.7_{787.0}^{794.3}$ & -24.9 & -25.2 \\
\hline 292 & 44.06 & $22.8_{22.5}^{23.0}$ & 0.618 & s & $10.8_{10.8}^{10.8}$ & $13.6_{13.6}^{13.6}$ & -19.4 & -20.6 \\
\hline 313 & 44.03 & $22.7_{21.9}^{23.0}$ & 0.970 & $\mathrm{p}$ & $10.9_{10.9}^{10.9}$ & $7.4_{7.4}^{7.5}$ & -20.0 & -21.2 \\
\hline 323 & 44.27 & $22.3_{22.1}^{22.4}$ & 0.839 & $\mathrm{~s}$ & $10.8_{10.8}^{10.8}$ & $60.0_{58.2}^{60.5}$ & -20.8 & -21.8 \\
\hline 327 & 44.31 & $22.9_{22.2}^{23.4}$ & 1.910 & $\mathrm{p}$ & $11.2_{11.2}^{11.2}$ & $220.8_{220.3}^{220.8}$ & -22.2 & -23.1 \\
\hline 331 & 44.50 & $22.3_{21.9}^{22.7}$ & 2.390 & $\mathrm{p}$ & $11.0_{10.9}^{11.1}$ & $89.5_{36.5}^{89.7}$ & -21.2 & -22.2 \\
\hline 333 & 44.74 & $23.2_{20.4}^{-1.0}$ & 3.070 & $\mathrm{p}$ & $11.0_{11.0}^{11.0}$ & $150.0_{149.6}^{150.0}$ & -23.0 & -23.7 \\
\hline 337 & 44.61 & $22.7_{22.4}^{22.9}$ & 1.720 & $\mathrm{p}$ & $10.9_{10.9}^{10.9}$ & $179.9_{177.8}^{180.7}$ & -21.3 & -22.2 \\
\hline 338 & 44.35 & $23.0_{22.4}^{23.4}$ & 1.550 & $\mathrm{p}$ & $11.0_{11.0}^{11.0}$ & $3.2_{3.2}^{3.2}$ & -19.9 & -21.1 \\
\hline 351 & 44.72 & $22.9_{20.4}^{-1.0}$ & 2.200 & $\mathrm{p}$ & $11.2_{11.2}^{11.2}$ & $17.7_{17.1}^{18.2}$ & -20.9 & -22.1 \\
\hline 362 & 44.22 & $22.8_{22.3}^{23.4}$ & 1.490 & $\mathrm{p}$ & $10.3_{10.2}^{10.2}$ & $4.6_{4.5}^{4.7}$ & -20.1 & -20.9 \\
\hline 390 & 44.67 & $22.8_{22.4}^{23.2}$ & 1.740 & $\mathrm{p}$ & $11.4_{11.4}^{11.4}$ & $98.4_{98.4}^{102.8}$ & -20.9 & -22.0 \\
\hline 403 & 44.59 & $22.2_{21.6}^{22.5}$ & 2.510 & $\mathrm{p}$ & $9.9_{9.9}^{9.9}$ & $12.5_{11.6}^{12.9}$ & -20.2 & -20.9 \\
\hline 406 & 44.68 & $23.0_{22.7}^{23.3}$ & 2.921 & $\mathrm{~s}$ & $10.9_{10.9}^{10.9}$ & $37.2_{36.9}^{37.0}$ & -21.7 & -22.5 \\
\hline 411 & 44.09 & $22.4_{21.9}^{22.6}$ & 0.952 & s & $10.8_{10.8}^{10.8}$ & $0.8_{0.8}^{0.8}$ & -20.1 & -21.3 \\
\hline 412 & 44.42 & $22.8_{22.7}^{23.0}$ & 1.300 & $\mathrm{p}$ & $10.6_{10.6}^{10.6}$ & $108.1_{107.6}^{109.1}$ & -20.7 & -21.6 \\
\hline 413 & 44.80 & $23.6_{23.3}^{23.9}$ & 1.023 & $\mathrm{~s}$ & $10.6_{10.6}^{10.6}$ & $47.2_{47.2}^{47.0}$ & -21.7 & -22.5 \\
\hline 428 & 44.52 & $22.2_{21.7}^{22.6}$ & 2.010 & $\mathrm{p}$ & $11.1_{11.1}^{11.0}$ & $1.0_{1.0}^{1.0}$ & -21.7 & -22.8 \\
\hline 435 & 44.49 & $22.5_{22.2}^{22.9}$ & 2.080 & $\mathrm{p}$ & $10.3_{10.3}^{10.3}$ & $32.9_{32.8}^{33.6}$ & -21.9 & -22.6 \\
\hline 440 & 44.25 & $22.3_{20.7}^{22.7}$ & 1.800 & $\mathrm{p}$ & $\begin{array}{r}11.0_{11.0}^{11.0} \\
\text { 1.0 }\end{array}$ & $98.2_{97.3}^{99.5}$ & -20.7 & -21.8 \\
\hline 463 & 44.32 & $22.6_{22.3}^{23.0}$ & 1.870 & $\mathrm{p}$ & $11.2_{11.2}^{11.2}$ & $3.4_{3.4}^{3.4}$ & -20.5 & -21.8 \\
\hline 470 & 44.31 & $22.7_{21.8}^{23.4}$ & 2.070 & $\mathrm{p}$ & $10.9_{10.9}^{10.2}$ & $1.7_{1.7}^{1.7}$ & -19.8 & -21.1 \\
\hline 2206 & 45.42 & $23.0_{22.9}^{23.0}$ & 2.410 & $\mathrm{p}$ & $\begin{array}{r}11.0_{11.0}^{11.0} \\
n_{1.0}\end{array}$ & $160.7_{160.0}^{161.4}$ & -21.8 & -22.8 \\
\hline 2208 & 45.08 & $23.0_{22.9}^{23.0}$ & 1.130 & $\mathrm{p}$ & $10.7_{10.6}^{10.7}$ & $75.0_{73.5}^{77.0}$ & -20.4 & -21.4 \\
\hline
\end{tabular}

Notes. The legend for the column named Survey is the following: s = spectroscopic redshift; $\mathrm{p}=$ photometric redshift. ${ }^{(10)}$ The photometry is contaminated by the halo of a bright nearby star. 
V. Mainieri et al.: Black hole accretion and host galaxies of obscured quasars in XMM-COSMOS

Table 1. continued.

\begin{tabular}{|c|c|c|c|c|c|c|c|c|}
\hline XID & $\begin{array}{c}\log L_{\mathrm{X}}[0.5-10 \mathrm{keV}] \\
\left(\mathrm{erg} \mathrm{s}^{-1}\right)\end{array}$ & $\begin{array}{l}\log N_{\mathrm{H}} \\
\left(\mathrm{cm}^{-2}\right) \\
\end{array}$ & $z$ & Survey & $\begin{array}{c}\log M \star \\
\left(M_{\odot}\right) \\
\end{array}$ & $\begin{array}{c}S F R \\
\left(M_{\odot} \mathrm{yr}^{-1}\right) \\
\end{array}$ & $\begin{array}{c}M_{U} \\
(\mathrm{AB})\end{array}$ & $\begin{array}{c}M_{B} \\
(\mathrm{AB}) \\
\end{array}$ \\
\hline 2210 & 44.08 & $22.8_{22.6}^{23.0}$ & 0.968 & $\mathrm{~s}$ & $11.0_{11.0}^{11.0}$ & $10.7_{10.6}^{10.7}$ & -20.9 & -22.1 \\
\hline 2236 & 44.70 & $22.0_{21 .}^{22.3}$ & 1.800 & $\mathrm{p}$ & $10.4_{10.4}^{10.0}$ & $289.1_{289.1}^{10.0}$ & -21.4 & -22.1 \\
\hline 2237 & 44.48 & $22.4_{22.3}^{22.6}$ & 0.944 & $\mathrm{~s}$ & $11.2_{11.2}^{11.4}$ & $17.7_{17.6}^{17.1}$ & -21.5 & -22.6 \\
\hline 2289 & 44.17 & $22.6_{22.3}^{22.8}$ & 0.833 & $\mathrm{~s}$ & $11.0_{110}^{11.0}$ & $114.6_{114.8}^{11.0}$ & -21.5 & -22.5 \\
\hline 2336 & 44.67 & $22.8_{22.5}^{23.3}$ & 1.760 & $\mathrm{p}$ & $\begin{array}{l}10.9_{10.9}^{11.9} \\
10.9\end{array}$ & $6.6_{6.4}^{6.6}$ & -20.9 & -21.9 \\
\hline $2390^{11}$ & 44.94 & $23.3_{231}^{23.6}$ & 2.830 & $\mathrm{p}$ & $10.6_{10.6}^{10.6}$ & $223.4_{220.4}^{224.9}$ & -23.8 & -24.2 \\
\hline 2408 & 44.24 & $23.0_{22.7}^{23.1}$ & 1.270 & $\mathrm{~s}$ & $11.1_{11.1}^{11.1}$ & $317.0_{317.0}^{317.0}$ & -21.9 & -22.8 \\
\hline 2414 & 44.55 & $22.2_{22.0}^{22.4}$ & 1.640 & $\mathrm{p}$ & $11.2_{11.2}^{11.2}$ & $68.4_{68.2}^{71.9}$ & -21.7 & -22.7 \\
\hline 2440 & 44.02 & $22.3_{22.0}^{22.5}$ & 1.175 & $\mathrm{~s}$ & $11.0_{11.0}^{11.1}$ & $0.8_{0.8}^{0.8}$ & -21.5 & -22.7 \\
\hline 2464 & 44.11 & $22.4_{22.2}^{22.6}$ & 1.260 & $\mathrm{p}$ & $\begin{array}{l}10.9_{10.9}^{10.9} \\
\text { (1) }\end{array}$ & $0.6_{0.6}^{0.6}$ & -21.2 & -22.4 \\
\hline 2483 & 44.62 & $22.4_{22.0}^{22.7}$ & 1.650 & $\mathrm{p}$ & $10.8_{10.7}^{10.8}$ & $98.6_{96.4}^{101.2}$ & -21.3 & -22.2 \\
\hline 2507 & 44.40 & $22.6_{22.2}^{22.9}$ & 0.937 & $\mathrm{~s}$ & $9.8_{9.8}^{9.8}$ & $86.3_{86.3}^{87.1}$ & -21.9 & -22.3 \\
\hline 2518 & 44.90 & $23.1_{22.7}^{23.4}$ & 3.176 & $\mathrm{~s}$ & $11.5_{11.5}^{11.5}$ & $77.4_{77.4}^{77.4}$ & -22.7 & -23.6 \\
\hline 2528 & 44.78 & $22.3_{21.8}^{22.6}$ & 2.050 & $\mathrm{p}$ & $9.9_{9.9}^{9.9}$ & $195.9_{194.5}^{197.2}$ & -22.0 & -22.4 \\
\hline 2530 & 45.02 & $22.4_{20.4}^{-1.0}$ & 2.640 & $\mathrm{p}$ & $11.8_{11.8}^{11.8}$ & $3026.9_{2992.3}^{3026.9}$ & -24.8 & -25.5 \\
\hline 2544 & 44.65 & $23.3_{23.0}^{23.6}$ & 0.826 & $\mathrm{~s}$ & $11.3_{11.3}^{11.3}$ & $2.6_{2.6}^{2.6}$ & -21.4 & -22.5 \\
\hline $2582^{12}$ & 44.25 & $22.5_{22.0}^{22.8}$ & 2.090 & $\mathrm{p}$ & $11.3_{11.2}^{11.3}$ & $0.0_{0.0}^{3.4}$ & -18.3 & -20.0 \\
\hline 2597 & 44.01 & $22.3_{21.8}^{22.6}$ & 1.119 & $\mathrm{~s}$ & $10.7_{10.7}^{10.7}$ & $23.4_{23.3}^{23.4}$ & -20.5 & -21.5 \\
\hline 2668 & 45.32 & $23.2_{20.4}^{23.6}$ & 0.886 & $\mathrm{~s}$ & $10.8_{10.8}^{10.8}$ & $0.4_{0.4}^{0.4}$ & -19.6 & -21.0 \\
\hline 2684 & 44.47 & $22.5_{22.2}^{22.7}$ & 1.810 & $\mathrm{p}$ & $11.1_{11.1}^{11.1}$ & $12.1_{12.1}^{12.1}$ & -20.9 & -22.0 \\
\hline 2772 & 44.65 & $22.4_{21.8}^{22.7}$ & 1.600 & $\mathrm{p}$ & $11.2_{11.2}^{11.2}$ & $1.1_{1.1}^{1.1}$ & -21.8 & -23.0 \\
\hline 5006 & 45.52 & $24.1_{23.8}^{24.0}$ & 2.417 & s & $11.3_{11.3}^{11.3}$ & $540.8_{538.3}^{543.3}$ & -22.5 & -23.4 \\
\hline 5007 & 44.99 & $23.8_{20.4}^{24.5}$ & 2.110 & $\mathrm{p}$ & $10.5_{10.5}^{10.6}$ & $21.9_{10.3}^{24.9}$ & -19.2 & -20.3 \\
\hline 5033 & 44.62 & $23.6_{23.6}^{24.6}$ & 0.670 & $\mathrm{p}$ & $10.7_{10.7}^{10.7}$ & $1.0_{1.0}^{1.0}$ & -20.3 & -21.4 \\
\hline 5039 & 44.40 & $22.5_{21.8}^{22.9}$ & 1.750 & $\mathrm{p}$ & $11.2_{11.2}^{11.2}$ & $81.7_{79.6}^{83.8}$ & -20.6 & -21.7 \\
\hline 5043 & 44.14 & $22.9_{20.4}^{24.0}$ & 1.380 & $\mathrm{p}$ & $10.8_{10.8}^{10.8}$ & $65.5_{65.5}^{65.0}$ & -20.3 & -21.4 \\
\hline 5053 & 44.30 & $23.0_{22.5}^{23.4}$ & 1.200 & $\mathrm{p}$ & $11.0_{11.0}^{11.0}$ & $0.8_{0.8}^{0.8}$ & -20.9 & -22.1 \\
\hline 5054 & 44.54 & $22.0_{21.7}^{22.3}$ & 1.420 & $\mathrm{p}$ & $10.8_{10.8}^{10.8}$ & $38.9^{39.5}$ & -19.8 & -21.0 \\
\hline 5056 & 44.19 & $22.5_{22.3}^{22.8}$ & 1.220 & $\mathrm{p}$ & $11.0_{11.0}^{11.0}$ & $0.7_{0.7}^{0.7}$ & -20.7 & -21.9 \\
\hline 5057 & 44.19 & $22.2_{20.8}^{22.7}$ & 1.690 & $\mathrm{p}$ & $11.1_{11.0}^{11.1}$ & $115.1_{115.1}^{116.7}$ & -21.5 & -22.5 \\
\hline 5066 & 44.42 & $22.4_{21.9}^{22.9}$ & 0.970 & $\mathrm{p}$ & $11.0_{11.0}^{11.0}$ & $9.5_{9.5}^{9.6}$ & -20.8 & -22.0 \\
\hline $5070^{13}$ & 44.77 & $23.2_{23.1}^{23.4}$ & 1.450 & $\mathrm{p}$ & $10.3_{10.3}^{10.3}$ & $462.4_{461.3}^{465.6}$ & -24.7 & -24.9 \\
\hline 5111 & 44.63 & $22.9_{22.6}^{23.1}$ & 1.080 & $\mathrm{p}$ & $10.0_{10.0}^{10.1}$ & $12.4_{12.4}^{12.4}$ & -19.7 & -20.6 \\
\hline 5120 & 44.98 & $22.4_{21.5}^{22.6}$ & 3.650 & $\mathrm{p}$ & $\ldots$ & $\ldots$ & 0.0 & 0.0 \\
\hline 5149 & 44.07 & $22.2_{20.4}^{22.8}$ & 1.560 & $\mathrm{p}$ & $10.6_{10.6}^{10.7}$ & $93.8_{87.5}^{94.6}$ & -20.6 & -21.5 \\
\hline 5153 & 44.13 & $22.9_{22.7}^{23.4}$ & 0.787 & $\mathrm{~s}$ & $10.0_{10.0}^{10.0}$ & $\begin{array}{r}47.1_{47.1}^{47.9} \\
\text { (1) }\end{array}$ & -19.6 & -20.5 \\
\hline 5158 & 44.22 & $22.4_{22.0}^{22.6}$ & 1.440 & $\mathrm{p}$ & $10.9_{10.9}^{10.9}$ & $22.9_{22.9}^{22.9}$ & -21.7 & -22.5 \\
\hline 5161 & 45.54 & $22.9_{20.4}^{23.7}$ & 3.160 & $\mathrm{p}$ & $11.1_{11.1}^{11.9}$ & $177.4_{175.8}^{2177.4}$ & -22.6 & -23.4 \\
\hline 5162 & 44.94 & $22.5_{20.4}^{22.4}$ & 3.524 & $\mathrm{~s}$ & $10.5_{10.5}^{11.5}$ & $699.8_{695.8}^{699.8}$ & -23.4 & -23.8 \\
\hline 5178 & 44.43 & $23.1_{22.8}^{23.4}$ & 1.720 & $\mathrm{p}$ & $11.3_{11.3}^{11.3}$ & $1.3_{1.3}^{1.3}$ & -21.4 & -22.7 \\
\hline 5186 & 45.16 & $22.6_{22.4}^{22.8}$ & 2.540 & $\mathrm{p}$ & $\begin{array}{l}9.9_{9.9}^{9.9} \\
\text { (1.) }\end{array}$ & $195.4_{188.8}^{195.4}$ & -20.8 & -21.4 \\
\hline 5229 & 44.37 & $22.6_{22.1}^{23.4}$ & 1.030 & $\mathrm{p}$ & $10.7_{10.8}^{10.8}$ & $11.1_{11.1}^{11.7}$ & -19.8 & -20.9 \\
\hline 5232 & 44.73 & $22.9_{218}^{23.0}$ & 2.830 & $\mathrm{p}$ & $11.2_{11.2}^{11.2}$ & $\begin{array}{l}72.8_{70.7}^{89.7} \\
\end{array}$ & -20.6 & -21.7 \\
\hline 5266 & 44.72 & $23.1_{228}^{23.8}$ & 1.950 & $\mathrm{p}$ & $10.8_{108}^{11.8}$ & $54.6_{546}^{56.8}$ & -20.7 & -21.7 \\
\hline 5278 & 44.94 & $23.2_{22.4}^{23.8}$ & 1.620 & $\mathrm{p}$ & $10.6_{106}^{10.8}$ & $32.6_{32.134 .0}^{32.0}$ & -20.7 & -21.6 \\
\hline 5288 & 44.15 & $22.2_{21.9}^{22.6}$ & 1.000 & $\mathrm{~s}$ & $11.1_{11.1}^{11.0}$ & $2.2_{2.2}^{2.2 .1}$ & -20.7 & -21.9 \\
\hline
\end{tabular}

Notes. ${ }^{(11)}$ The ACS image shows a source at $0.5^{\prime \prime}$ from the chosen counterpart, therefore the IR photometry is the blending of the emission of these two sources. ${ }^{(12)}$ For this objects we have photometry only in five filters. ${ }^{(13)}$ The photometry is contaminated by a nearby bright star. 
Table 1. continued.

\begin{tabular}{|c|c|c|c|c|c|c|c|c|}
\hline XID & $\begin{array}{c}\log L_{\mathrm{X}}[0.5-10 \mathrm{keV}] \\
\left(\mathrm{erg} \mathrm{s}^{-1}\right)\end{array}$ & $\begin{array}{l}\log N_{\mathrm{H}} \\
\left(\mathrm{cm}^{-2}\right) \\
\end{array}$ & $z$ & Survey & $\begin{array}{c}\log M \star \\
\left(M_{\odot}\right) \\
\end{array}$ & $\begin{array}{c}S F R \\
\left(M_{\odot} \mathrm{yr}^{-1}\right)\end{array}$ & $\begin{array}{c}M_{U} \\
(\mathrm{AB})\end{array}$ & $\begin{array}{c}M_{B} \\
(\mathrm{AB}) \\
\end{array}$ \\
\hline 5292 & 44.57 & $22.4_{20.4}^{23.0}$ & 2.730 & $\mathrm{p}$ & $11.0_{109}^{11.0}$ & $27.0_{27.0}^{37.3}$ & -19.7 & -21.0 \\
\hline $5305^{14}$ & 44.17 & $22.4_{21.4}^{20.4}$ & 2.500 & $\mathrm{p}$ & $10.1_{10.1}^{10.9}$ & $161.8_{158.19}^{164.1}$ & -21.4 & -21.9 \\
\hline $5326^{15}$ & 44.35 & $22.7_{220}^{21.4}$ & 0.950 & $\mathrm{p}$ & $8.6_{86}^{8.6 .1}$ & $9.2_{92}^{9.3 .9}$ & -19.3 & -19.6 \\
\hline 5347 & 44.65 & $23.1_{204}^{24.3}$ & 3.120 & p & $11.4_{11.4}^{11.0}$ & $212.8_{208.9}^{224.9}$ & -22.2 & -23.2 \\
\hline 5357 & 44.83 & $23.6_{23.9}^{20.4}$ & 1.670 & $\mathrm{p}$ & $\begin{array}{r}10.7_{107}^{11.4} \\
107\end{array}$ & $1.6_{16}^{1.7}$ & -20.4 & -21.4 \\
\hline 5368 & 44.17 & $22.2_{219}^{22.4}$ & 1.230 & p & $11.2_{11.2}^{10.1}$ & $105.9_{1059}^{105.9}$ & -20.9 & -22.1 \\
\hline 5369 & 44.16 & $22.1_{218}^{22.4}$ & 0.950 & $\mathrm{p}$ & $10.4_{104}^{11.2}$ & $21.1_{210}^{21.9}$ & -19.7 & -20.7 \\
\hline 5400 & 44.19 & $22.7_{22.9}^{21.8}$ & 0.680 & p & $\begin{array}{l}10.8_{10.8}^{10.4} \\
\end{array}$ & $1.2_{1.2}^{1.2}$ & -20.0 & -21.2 \\
\hline 5414 & 44.10 & $22.4_{222}^{22.5}$ & 0.910 & p & $10.7_{10.7}^{10.8}$ & $\begin{array}{l}1.2 \\
0.9^{1.09}\end{array}$ & -19.7 & -20.9 \\
\hline 5417 & 44.62 & $22.4_{219}^{22.8}$ & 2.050 & $\mathrm{p}$ & $11.0_{11.0}^{10.1}$ & $1153.5_{11535}^{1153.5}$ & -24.1 & -24.6 \\
\hline 5427 & 44.86 & $23.6_{234}^{21.9}$ & 1.177 & $\mathrm{~s}$ & $11.1_{11.1}^{11.0}$ & $2.7^{2.7}$ & -21.5 & -22.6 \\
\hline 5441 & 44.11 & $22.1_{217}^{23.5}$ & 1.170 & $\mathrm{p}$ & $11.1_{11.1}^{11.1}$ & $4.2_{42}^{4.2}$ & -20.8 & -21.9 \\
\hline 5448 & 45.30 & $22.8_{225}^{21.7}$ & 2.060 & $\mathrm{p}$ & $11.0_{110}^{11.0}$ & $43.0_{42.8}^{43.2}$ & -21.8 & -22.7 \\
\hline 5452 & 44.40 & $22.0_{21.6}^{22.4}$ & 1.920 & $\mathrm{p}$ & $10.0_{10.0}^{10.0}$ & $131.5_{131.5}^{42.0}$ & -21.2 & -21.7 \\
\hline 5467 & 44.14 & $23.2_{20.4}^{21.6}$ & 0.870 & $\mathrm{p}$ & $10.7_{10.7}^{10.0}$ & $1.6_{16}^{1.6}$ & -19.1 & -20.3 \\
\hline 5488 & 44.36 & $23.1_{22.2}^{23.6}$ & 1.280 & $\mathrm{p}$ & $11.0_{11.0}^{11.7}$ & $163.7_{163.3}^{1604.4}$ & -21.8 & -22.8 \\
\hline 5496 & 44.60 & $23.6_{23.6}^{25.2}$ & 0.694 & $\mathrm{~s}$ & $10.6_{106}^{110.0}$ & $54.5_{54.5}^{54.3}$ & -20.6 & -21.6 \\
\hline 5512 & 44.31 & $22.1_{20.6}^{22.6}$ & 1.130 & $\mathrm{p}$ & $11.4_{11.4}^{11.4}$ & $5.2_{5.1}^{5.2}$ & -22.2 & -23.3 \\
\hline 5546 & 44.62 & $22.8_{20.4}^{-1.0}$ & 1.200 & $\mathrm{p}$ & $11.1_{11.1}^{11.4}$ & $2.4_{2.4}^{2.4}$ & -21.3 & -22.5 \\
\hline 5547 & 44.07 & $22.4_{22.6}^{22.4}$ & 0.960 & $\mathrm{p}$ & $9.5_{9.5}^{9.5}$ & $17.5_{17.5}^{2.4}$ & -19.2 & -19.9 \\
\hline 5548 & 45.01 & $23.1_{22.8}^{23.5}$ & 1.060 & $\mathrm{p}$ & $10.9_{10.9}^{10.9}$ & $1.0_{1.0}^{1.0}$ & -20.3 & -21.5 \\
\hline 5553 & 44.51 & $23.1_{20.4}^{-1.0}$ & 2.390 & $\mathrm{p}$ & $11.2_{11.1}^{11.2}$ & $1.1_{0.9}^{1.1}$ & -20.6 & -22.0 \\
\hline 5563 & 44.88 & $22.8_{22.6}^{23.4}$ & 3.410 & $\mathrm{p}$ & $10.3_{10.3}^{10.3}$ & $473.2_{443.6}^{493.2}$ & -21.7 & -22.3 \\
\hline 5569 & 45.04 & $22.7_{22.0}^{23.0}$ & 1.810 & $\mathrm{p}$ & $11.2_{11.2}^{11.2}$ & $3.3_{3.3}^{3.3 .0}$ & -21.1 & -22.3 \\
\hline 5573 & 44.88 & $22.2_{22.0}^{22.4}$ & 1.660 & $\mathrm{p}$ & $11.2_{11.2}^{11.2}$ & $182.0_{181.1}^{188.8}$ & -21.4 & -22.5 \\
\hline 5582 & 44.60 & $22.9_{22.7}^{23.1}$ & 1.490 & $\mathrm{p}$ & $10.7_{10.7}^{10.7}$ & $0.9_{0.9}^{0.9}$ & -20.3 & -21.4 \\
\hline 5606 & 44.73 & $22.6_{20.8}^{23.1}$ & 4.166 & $\mathrm{~s}$ & $10.2_{10.2}^{10.2}$ & $401.8_{399.0}^{401.8}$ & -23.4 & -23.7 \\
\hline 10094 & 44.69 & $22.9_{20.4}^{23.0}$ & 2.670 & $\mathrm{p}$ & $10.7_{10.7}^{10.7}$ & $5.0_{5.0}^{5.0}$ & -20.7 & -21.8 \\
\hline 10499 & 44.11 & $22.5_{21.7}^{23.0}$ & 1.990 & $\mathrm{p}$ & $11.2_{11.2}^{11.2}$ & $98.9_{98.9}^{98.9}$ & -20.9 & -22.0 \\
\hline 10690 & 44.52 & $22.7_{21.9}^{23.1}$ & 3.100 & $\mathrm{p}$ & $10.2_{10.2}^{10.2}$ & $177.8_{176.2}^{179.1}$ & -22.7 & -23.1 \\
\hline 10719 & 44.68 & $22.5_{22.1}^{22.9}$ & 1.700 & $\mathrm{p}$ & $10.5_{10.5}^{10.5}$ & $360.6_{360.6}^{364.2}$ & -22.9 & -23.3 \\
\hline 30292 & 44.37 & $23.0_{20.9}^{-1.0}$ & 2.550 & $\mathrm{p}$ & $10.0_{10.0}^{10.0}$ & $1.1_{1.1}^{1.1 .0}$ & -19.7 & -20.6 \\
\hline 30361 & 44.19 & $22.8_{20.4}^{23.4}$ & 1.910 & $\mathrm{p}$ & $11.1_{11.1}^{11.0}$ & $156.0_{151.4}^{156.0}$ & -21.2 & -22.2 \\
\hline 30789 & 44.99 & $22.8_{20.4}^{23.3}$ & 1.810 & $\mathrm{p}$ & $11.1_{11.1}^{11.1}$ & $\begin{array}{l}2.7_{2.7}^{2.7} \\
\text {. }\end{array}$ & -20.9 & -22.1 \\
\hline 31357 & 44.63 & $23.2_{22.9}^{20.5}$ & 1.700 & $\mathrm{p}$ & $11.4_{11.4}^{11.4}$ & $1.7_{1.7}^{1.1}$ & -21.7 & -23.0 \\
\hline 31419 & 44.35 & $23.6_{20.4}^{-1.9}$ & 1.130 & $\mathrm{p}$ & $9.9_{9.9}^{110.4}$ & $14.6_{70}^{14.7}$ & -18.6 & -19.6 \\
\hline 53351 & 45.80 & $23.2_{20.0}^{20.4}$ & 3.000 & $\mathrm{p}$ & $10.3_{101}^{10.3}$ & $53.7_{53.2}^{66.5}$ & -21.1 & -21.9 \\
\hline 54316 & 44.26 & $22.5_{22.9}^{20.4}$ & 1.980 & $\mathrm{p}$ & $11.2_{11.0}^{10.1}$ & $68.7_{68.7}^{16.2}$ & -21.1 & -22.2 \\
\hline 54514 & 44.44 & $23.6_{20.4}^{-1.0}$ & 0.707 & $\mathrm{~s}$ & $11.1_{11.1}^{11.0}$ & $2.5_{2.5}^{2.1}$ & -20.8 & -22.0 \\
\hline 60075 & 44.15 & $22.9_{22.4}^{23.4}$ & 1.080 & $\mathrm{p}$ & $10.8_{10.8}^{11.1}$ & $1.3_{1.3}^{1.3}$ & -19.4 & -20.7 \\
\hline 60144 & 44.07 & $23.0_{204}^{-1.4}$ & 1.500 & p & $\begin{array}{l}10.9_{10.9}^{10.8} \\
0\end{array}$ & $0.5_{0.5}^{0.5}$ & -21.1 & -22.2 \\
\hline 60168 & 44.84 & $22.6_{22.3}^{22.4}$ & 2.140 & $\mathrm{p}$ & $\begin{array}{l}10.9_{109}^{10.9} \\
\end{array}$ & $380.2_{372.4}^{383.7}$ & -21.9 & -22.7 \\
\hline 60214 & 44.17 & $22.8_{204}^{-1.0}$ & 1.640 & p & $\begin{array}{r}10.9 \\
10.9_{10.9}^{10.9}\end{array}$ & $0.3^{0.5}$ & -19.8 & -21.1 \\
\hline 60255 & 44.15 & $22.8_{204}^{-1.4}$ & 0.890 & p & $11.2_{11.2}^{10.8}$ & $161.8_{161.8}^{161.8}$ & -21.3 & -22.3 \\
\hline 60314 & 44.59 & $23.5_{204}^{20.4}$ & 1.080 & $\mathrm{p}$ & $10.3_{103}^{11.2 .3}$ & $49.8_{49.8}^{50.8}$ & -19.9 & -20.8 \\
\hline 60421 & 44.24 & $22.6_{204}^{20.4}$ & 1.600 & p & $10.0_{98}^{10.0}$ & $8.3_{80}^{18.5}$ & -19.9 & -20.7 \\
\hline 60422 & 45.17 & $23.0_{226}^{20.4}$ & 1.890 & $\mathrm{p}$ & $9.8_{9.8}^{9.8}$ & $71.3_{710}^{71.8}$ & -21.1 & -21.6 \\
\hline 60423 & 44.34 & $22.2_{204}^{22.8}$ & 1.560 & p & $10.9^{10.9}$ & $387.3_{387.3}^{393.6}$ & -22.6 & -23.3 \\
\hline
\end{tabular}

Notes. ${ }^{(14)}$ The quality of the SED fit is poor. ${ }^{(15)}$ The IR photometry is contaminated by a nearby source. 
V. Mainieri et al.: Black hole accretion and host galaxies of obscured quasars in XMM-COSMOS

Table 2. Morphological parameters of Type-2 QSO hosts at $z \leq 1.2$.

\begin{tabular}{|c|c|c|c|c|c|c|c|c|c|c|}
\hline XID & $z$ & $\begin{array}{c}\text { magI } \\
\mathrm{AB}\end{array}$ & Class & $\begin{array}{l}r_{1 / 2}{ }^{16} \\
\text { pixels }\end{array}$ & $\begin{array}{c}R_{\mathrm{P}} \\
\text { pixels }\end{array}$ & $\epsilon$ & $\mathrm{C}$ & A & G & $M_{20}$ \\
\hline 41 & 0.962 & 21.73 & 1 & 3.01 & 6.01 & 0.10 & 2.99 & 0.20 & 0.53 & -1.70 \\
\hline 70 & 0.688 & 20.51 & 2 & 15.14 & 30.35 & 0.28 & 2.42 & 0.15 & 0.47 & -1.66 \\
\hline 151 & 0.797 & 21.99 & 3 & 21.17 & 51.81 & 0.42 & 2.36 & 0.45 & 0.58 & -0.66 \\
\hline 200 & 1.156 & 23.34 & 3 & 11.18 & 31.52 & 0.25 & 3.24 & 0.31 & 0.49 & -1.89 \\
\hline 211 & 1.188 & 23.94 & 1 & 4.91 & 13.06 & 0.01 & 2.99 & 0.10 & 0.60 & -1.83 \\
\hline 212 & 0.931 & 22.47 & 1 & 7.73 & 19.87 & 0.17 & 3.16 & 0.07 & 0.60 & -2.10 \\
\hline 229 & 0.864 & 21.67 & 1 & 8.23 & 18.33 & 0.20 & 2.72 & 0.06 & 0.55 & -1.92 \\
\hline 292 & 0.618 & 21.68 & 2 & 21.47 & 41.24 & 0.67 & 2.45 & 0.18 & 0.53 & -1.79 \\
\hline 313 & 0.970 & 22.51 & 2 & 10.03 & 22.59 & 0.40 & 2.77 & 0.12 & 0.53 & -1.84 \\
\hline 323 & 0.839 & 21.29 & 2 & 16.77 & 36.11 & 0.56 & 3.49 & 0.15 & 0.51 & -2.29 \\
\hline 411 & 0.952 & 22.56 & 1 & 6.52 & 15.84 & 0.23 & 2.95 & 0.08 & 0.54 & -1.98 \\
\hline 413 & 1.023 & 21.46 & 3 & 31.00 & 83.23 & 0.31 & 3.51 & 0.39 & 0.50 & -1.86 \\
\hline 2208 & 1.130 & 23.04 & 1 & 7.99 & 21.86 & 0.25 & 3.35 & 0.23 & 0.58 & -1.90 \\
\hline 2210 & 0.968 & 21.48 & 1 & 13.38 & 35.99 & 0.18 & 3.75 & 0.10 & 0.57 & -2.28 \\
\hline 2237 & 0.944 & 20.94 & 2 & 12.57 & 34.64 & 0.44 & 3.97 & 0.14 & 0.58 & -2.33 \\
\hline 2289 & 0.833 & 20.79 & 1 & 6.68 & 15.90 & 0.29 & 3.69 & 0.09 & 0.61 & -2.18 \\
\hline 2440 & 1.175 & 22.27 & 1 & 9.62 & 22.94 & 0.19 & 3.05 & 0.12 & 0.58 & -1.92 \\
\hline 2597 & 1.119 & 22.89 & 1 & 9.60 & 29.59 & 0.20 & 3.52 & 0.20 & 0.60 & -2.06 \\
\hline 5033 & 0.670 & 21.02 & 3 & 18.95 & 39.91 & 0.20 & 1.99 & 0.21 & 0.53 & -1.17 \\
\hline 5053 & 1.200 & 23.83 & 1 & 8.78 & 29.95 & 0.29 & 3.25 & 0.24 & 0.53 & -2.06 \\
\hline 5229 & 1.030 & 23.32 & 1 & 6.46 & 14.65 & 0.23 & 2.95 & 0.13 & 0.56 & -1.90 \\
\hline 5288 & 1.000 & 21.98 & 1 & 7.52 & 18.34 & 0.26 & 3.09 & 0.06 & 0.57 & -1.96 \\
\hline 5326 & 0.950 & 23.97 & 3 & 7.76 & 17.84 & 0.52 & 2.69 & 0.32 & 0.56 & -0.99 \\
\hline 5400 & 0.680 & 21.18 & 1 & 10.04 & 23.91 & 0.20 & 3.42 & 0.07 & 0.62 & -2.18 \\
\hline 5414 & 0.910 & 22.59 & 1 & 8.60 & 19.75 & 0.46 & 2.75 & 0.09 & 0.54 & -1.81 \\
\hline 5427 & 1.177 & 22.13 & 2 & 18.08 & 45.91 & 0.26 & 3.24 & 0.21 & 0.54 & -2.11 \\
\hline 5441 & 1.170 & 22.77 & 1 & 14.84 & 38.39 & 0.30 & 3.81 & 0.24 & 0.51 & -2.16 \\
\hline 5467 & 0.870 & 23.13 & 1 & 6.63 & 15.94 & 0.11 & 2.94 & 0.11 & 0.57 & -1.94 \\
\hline 5496 & 0.694 & 21.06 & 3 & 12.10 & 27.63 & 0.41 & 3.28 & 0.23 & 0.59 & -1.84 \\
\hline 5546 & 1.200 & 22.25 & 1 & 4.16 & 8.85 & 0.10 & 2.80 & 0.14 & 0.56 & -1.81 \\
\hline 5547 & 0.960 & 23.77 & 2 & 6.37 & 20.50 & 0.15 & 3.75 & 0.20 & 0.59 & -2.14 \\
\hline 5548 & 1.060 & 22.77 & 2 & 13.83 & 30.79 & 0.48 & 3.21 & 0.16 & 0.53 & -2.14 \\
\hline 54514 & 0.707 & 20.41 & 1 & 13.76 & 42.68 & 0.32 & 3.72 & 0.11 & 0.66 & -2.12 \\
\hline 60314 & 1.080 & 23.26 & 1 & 6.51 & 15.56 & 0.24 & 2.69 & 0.13 & 0.55 & -1.81 \\
\hline
\end{tabular}

Notes. The morphological classes in Col. 4 are: $1=$ bulge dominated; $2=$ disk dominated; $3=$ possible merger. ${ }^{(16)}$ Half-light radius $\left(r_{1 / 2}\right)$ and Petrosian radius $\left(R_{\mathrm{P}}\right)$ are expressed in pixels, where $1 \mathrm{pxl}=0.03^{\prime \prime}$. 


\section{Appendix A: SED fits}

SED decomposition for the sample of Type-2 QSOs. Black circles are the observed photometry in the rest-frame. Purple and blue lines correspond respectively to the galaxy and AGN template found as the best fit solution, while the black line shows their sum. The triangle is the predicted AGN emission at $12.3 \mu \mathrm{m}$ based on the Gandhi et al. (2009) correlation. We tried to anchor the AGN template to this value allowing an uncertainty egual to the dispersion observed by Gandhi et al. (2009) in their correlation.
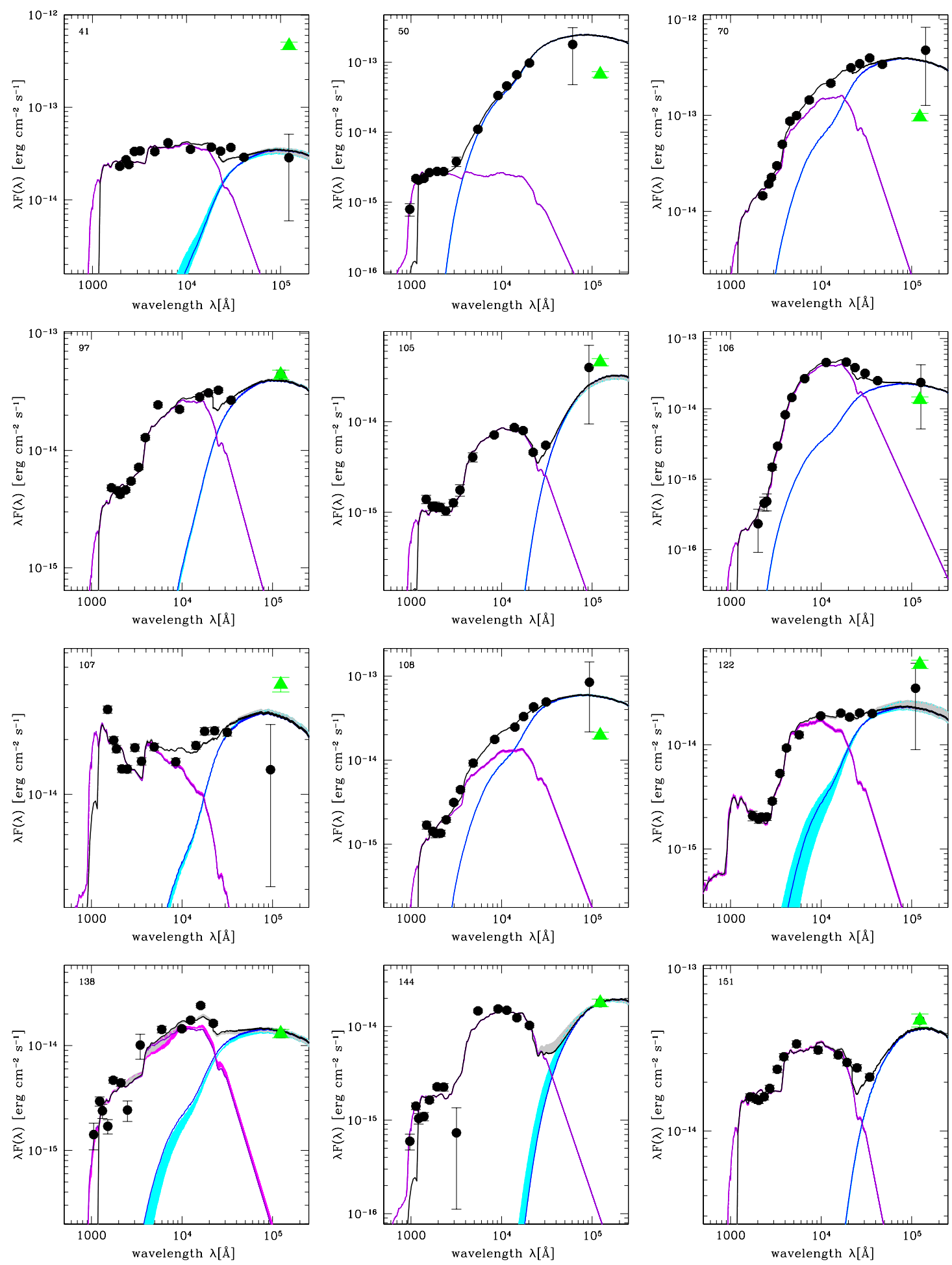
V. Mainieri et al.: Black hole accretion and host galaxies of obscured quasars in XMM-COSMOS
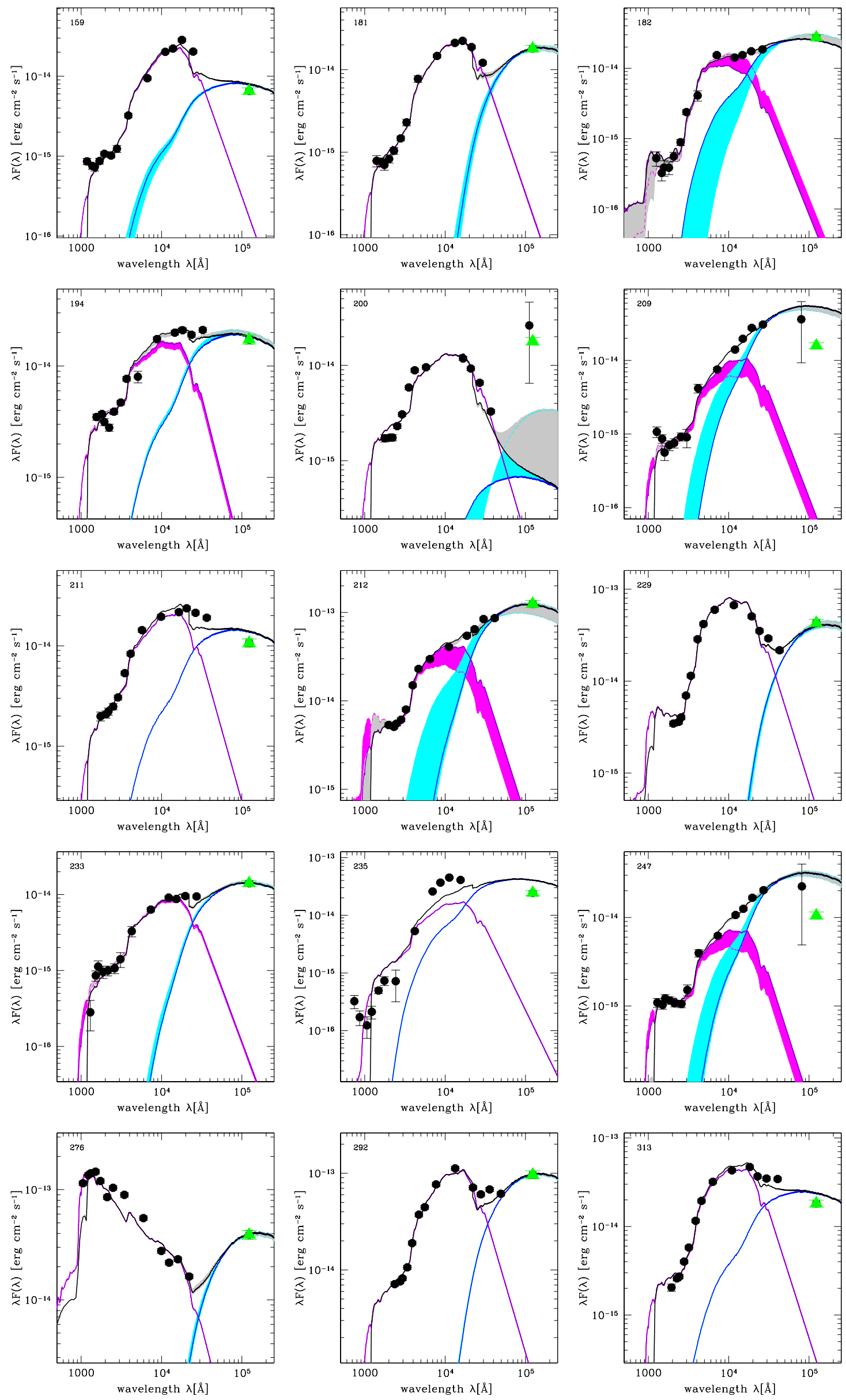

A80, page 19 of 27 
A\&A 535, A80 (2011)
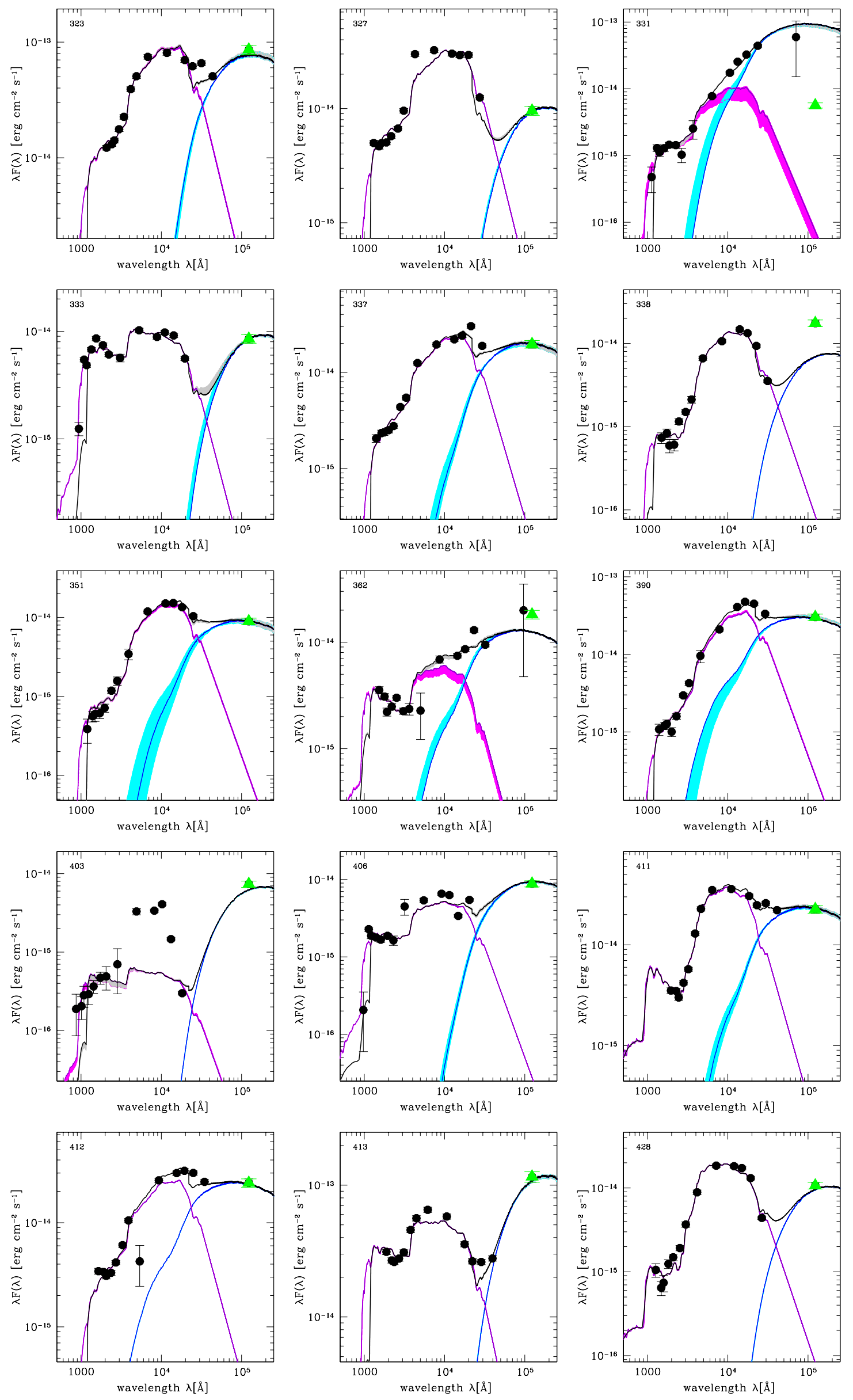
V. Mainieri et al.: Black hole accretion and host galaxies of obscured quasars in XMM-COSMOS
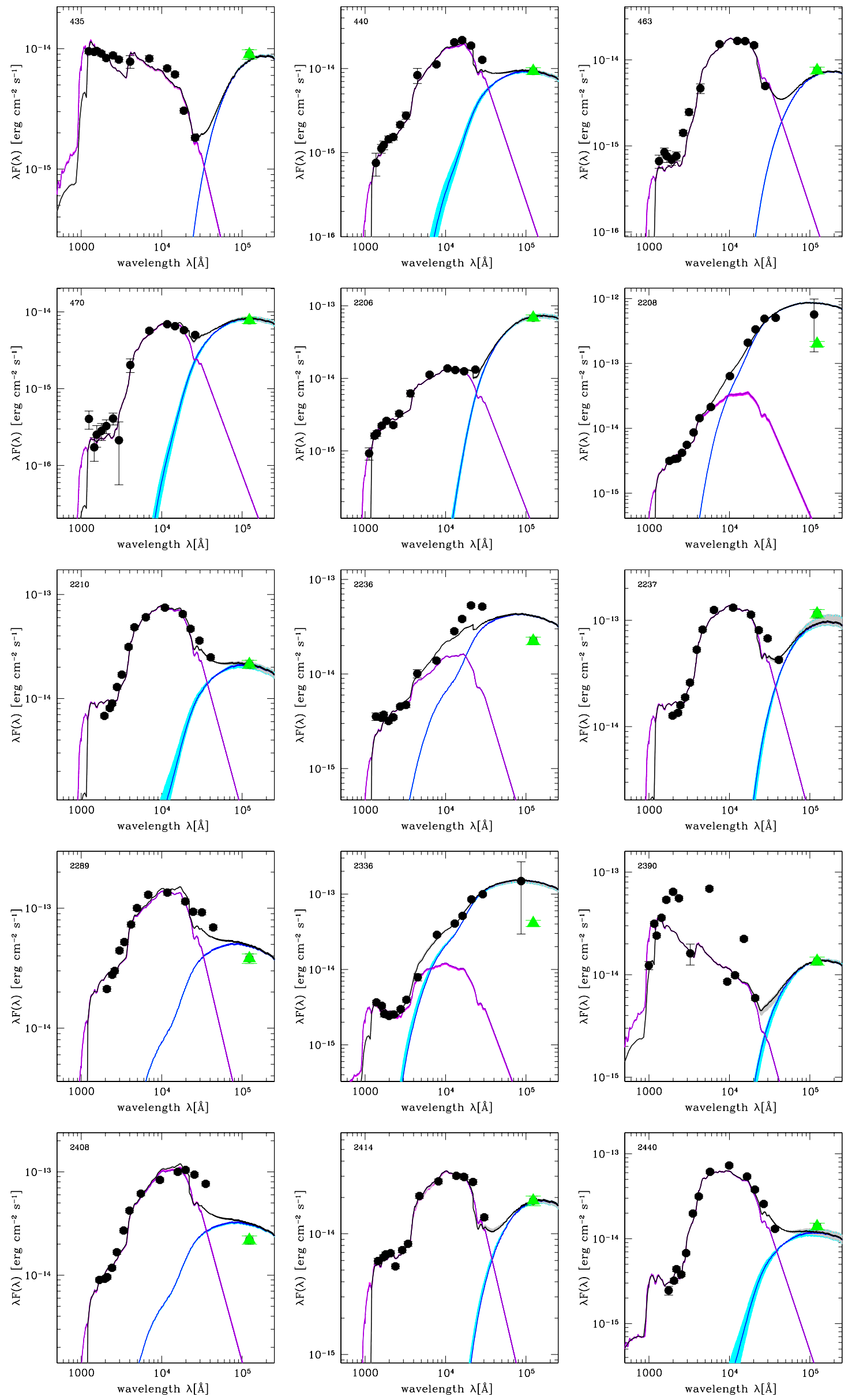

A80, page 21 of 27 
A\&A 535, A80 (2011)
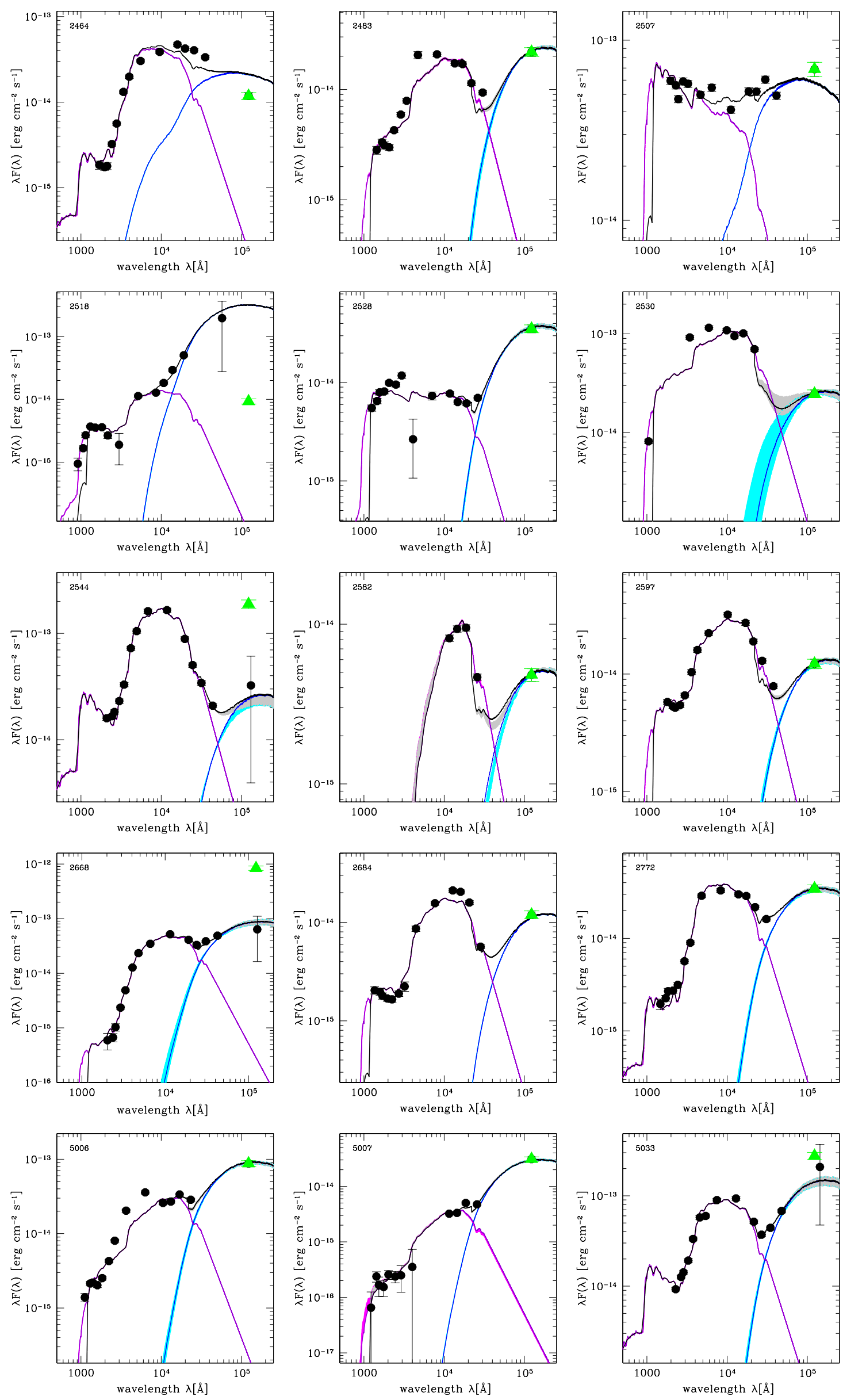
V. Mainieri et al.: Black hole accretion and host galaxies of obscured quasars in XMM-COSMOS
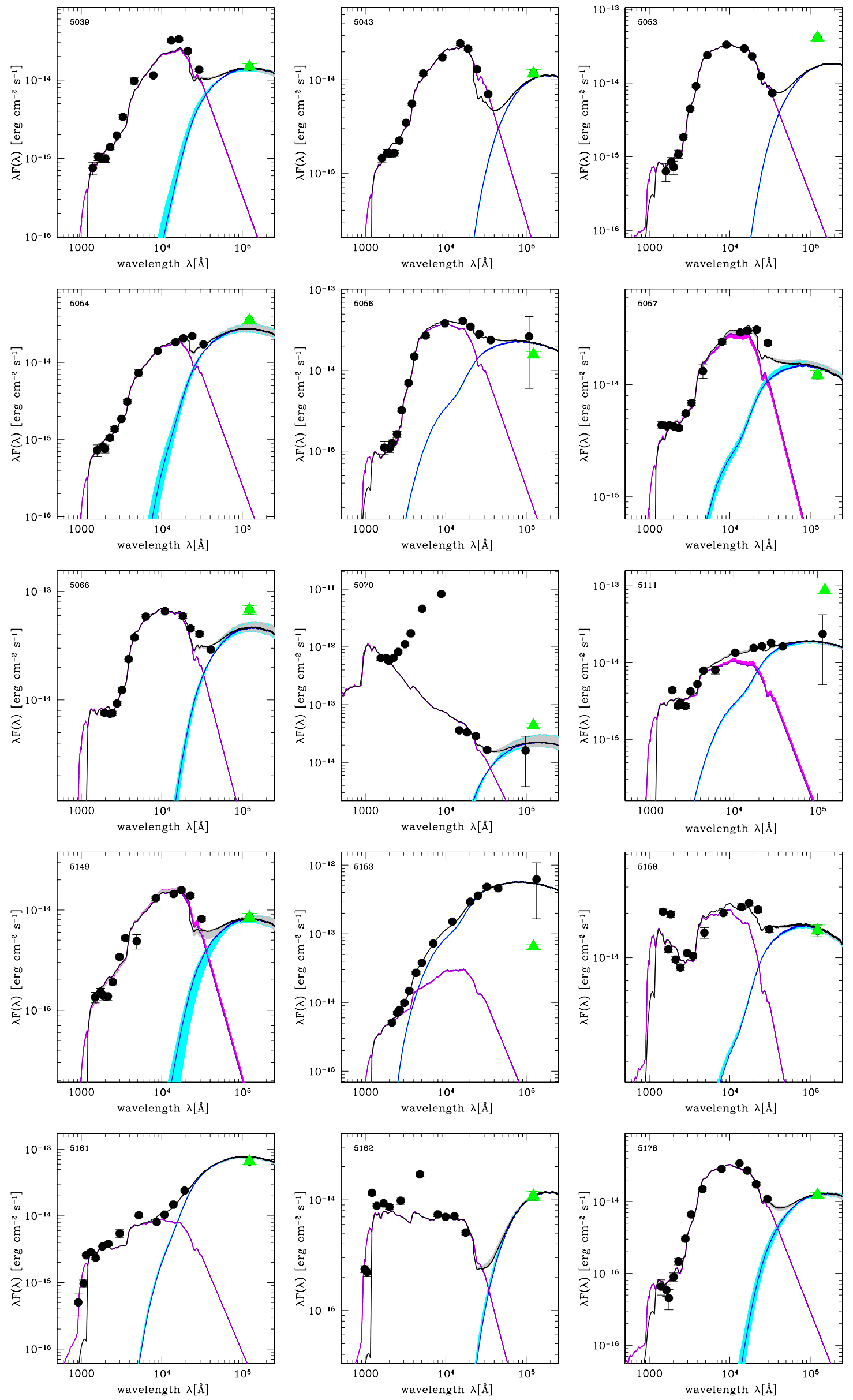

A80, page 23 of 27 
A\&A 535, A80 (2011)
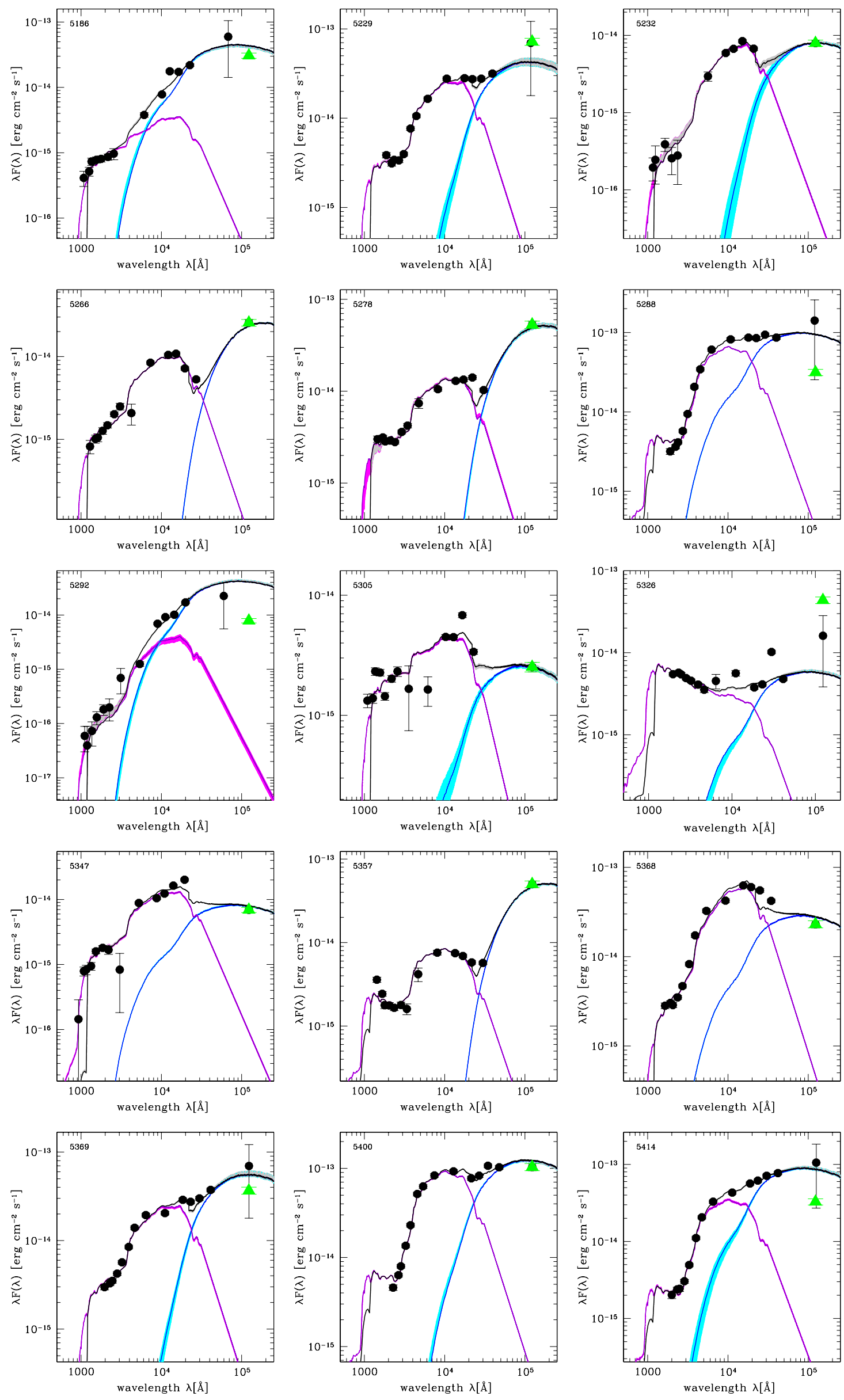
V. Mainieri et al.: Black hole accretion and host galaxies of obscured quasars in XMM-COSMOS
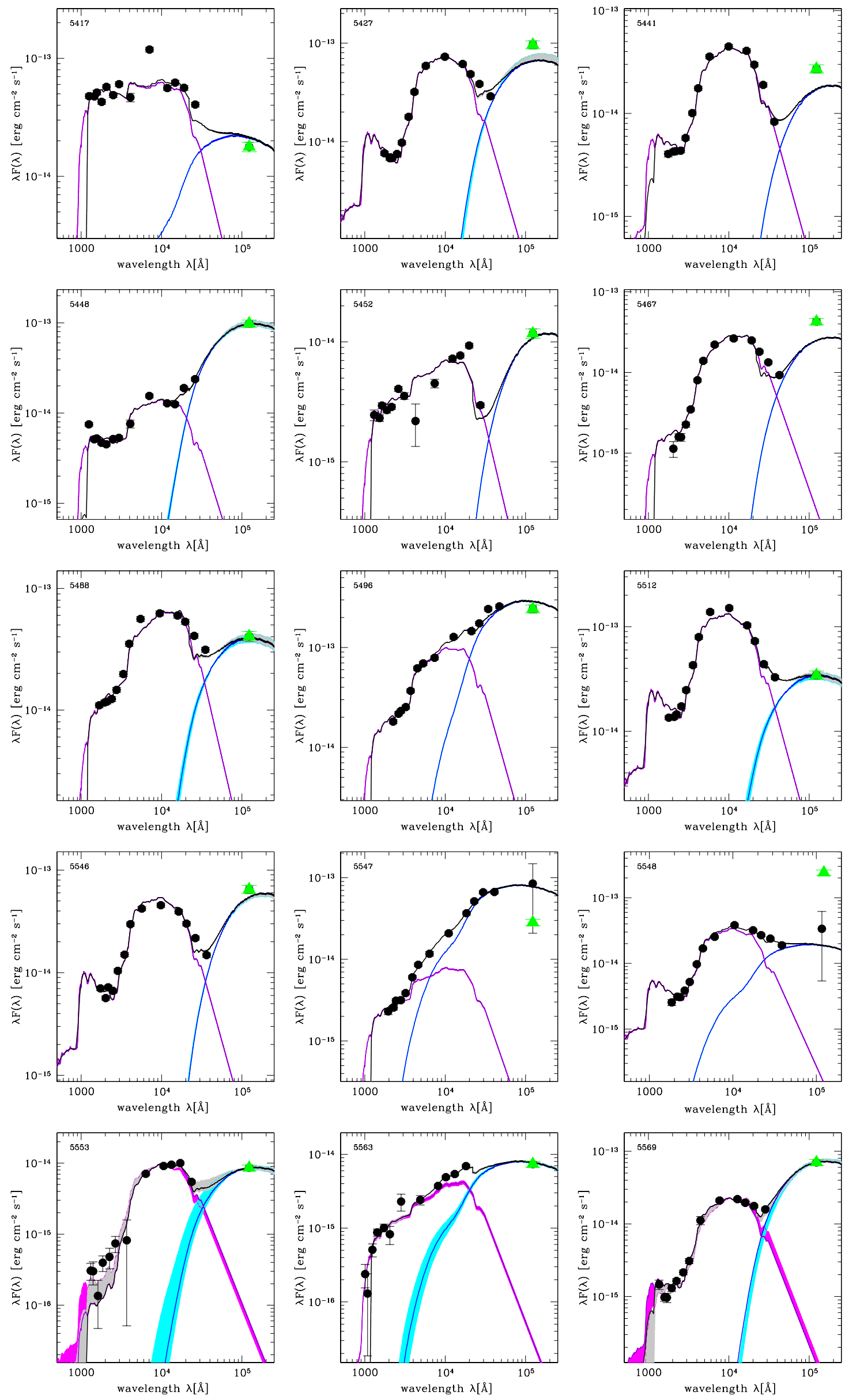

A80, page 25 of 27 
A\&A 535, A80 (2011)
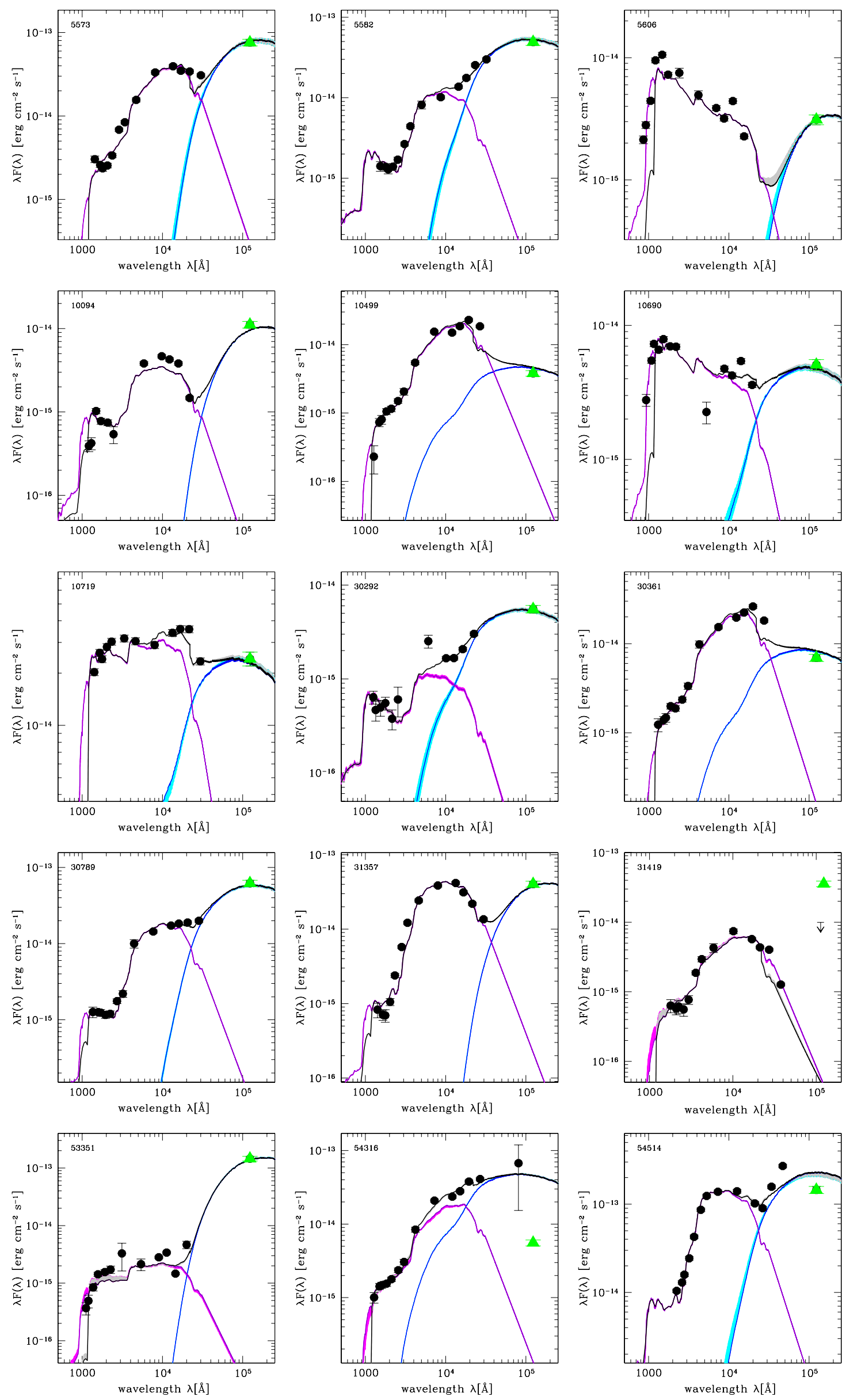
V. Mainieri et al.: Black hole accretion and host galaxies of obscured quasars in XMM-COSMOS
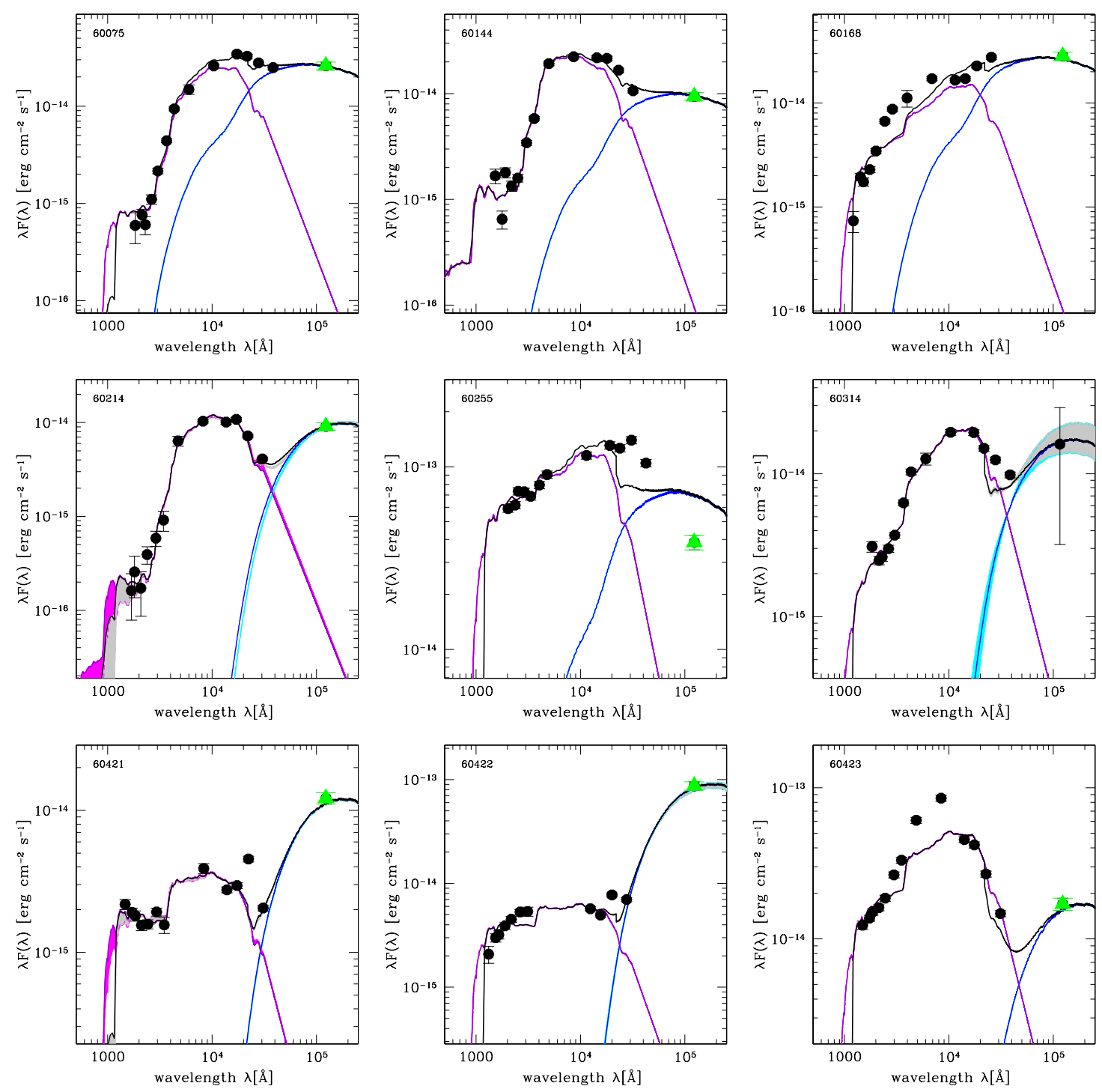